\title{
ON THE NON-COMMUTATIVE MAIN CONJECTURE FOR ELLIPTIC CURVES WITH COMPLEX MULTIPLICATION*
}

\author{
THANASIS BOUGANIS ${ }^{\dagger}$ AND OTMAR VENJAKOB ${ }^{\ddagger}$
}

Key words. (non-commutative) Iwasawa theory, Complex Multiplication, $K_{1}$ of Iwasawa algebras.

AMS subject classifications. Primary 11R23; Secondary 11S40, 14H52, 14K22, 19B28

In [7] a non-commutative Iwasawa Main Conjecture for elliptic curves over $\mathbb{Q}$ has been formulated. In this note we show that it holds for all CM-elliptic curves $E$ defined over $\mathbb{Q}$. This was claimed in (loc. cit.) without proof, which we want to provide now assuming that the torsion conjecture holds in this case. Based on this we show firstly the existence of the (non-commutative) $p$-adic $L$-function of $E$ and secondly that the (non-commutative) Main Conjecture follows from the existence of the Katz-measure, the work of Yager and Rubin's proof of the 2-variable main conjecture. The main issues are the comparison of the involved periods and to show that the (non-commutative) $p$-adic $L$-function is defined over the conjectured in (loc. cit.) coefficient ring. Moreover we generalize our considerations to the case of CMelliptic cusp forms.

Acknowledgements. We are grateful to John Coates and Sujatha for their interest and various discussions.

1. The non-commutative Main Conjecture. Let $E$ be an elliptic curve defined over $\mathbb{Q}$ and $p \geq 5$ a prime at which $E$ has good ordinary reduction. Then the $p$-adic Lie extension $F_{\infty}:=\mathbb{Q}(E(p))$ with Galois group $\mathcal{G}=G\left(F_{\infty} / \mathbb{Q}\right)$ contains the cyclotomic $\mathbb{Z}_{p}$-extension $\mathbb{Q}_{c y c}$ of $\mathbb{Q}$ and the quotient of $\mathcal{G}$ by its closed normal subgroup $\mathcal{H}:=G\left(F_{\infty} / \mathbb{Q}_{c y c}\right)$ equals $\Gamma:=G\left(\mathbb{Q}_{c y c} / \mathbb{Q}\right) \cong \mathbb{Z}_{p}$.

We write $D$ for the ring of integers $\mathcal{O}_{L}$, where $L$ is either a finite extension of $\mathbb{Q}_{p}$ or of the completion $\widehat{\mathbb{Q}_{p}^{n r}}$ of the maximal unramified extension $\mathbb{Q}_{p}^{n r}$ of $\mathbb{Q}_{p}$. As usual we write

$$
\Lambda:=\Lambda_{D}(\mathcal{G}):=D \llbracket \mathcal{G} \rrbracket
$$

for the Iwasawa algebra of $\mathcal{G}$ with coefficients in $D$. Note that is a Noetherian pseudocompact semi-local ring (which is compact if $L$ is finite over $\mathbb{Q}_{p}$ ). We denote by

$$
\mathfrak{M}_{\mathcal{H}}(\mathcal{G}):=\mathfrak{M}_{D, \mathcal{H}}(\mathcal{G})
$$

the category of all finitely $\Lambda(\mathcal{G})$-modules $M$ such that its quotient $M / M(p)$ by its $p$ primary subgroup $M(p)$ is finitely generated over the subalgebra $\Lambda(\mathcal{H})$ of $\Lambda(\mathcal{G})$. Also we recall from [7] the definition of the multiplicatively closed subsets

$$
S:=\{\lambda \in \Lambda \mid \Lambda / \Lambda \lambda \text { is finitely generated over } \Lambda(\mathcal{H})\}
$$

\footnotetext{
${ }^{*}$ Received August 5, 2010; accepted for publication August 20, 2010. The authors are supported by the ERC and DFG.

${ }^{\dagger}$ Universität Heidelberg, Mathematisches Institut, Im Neuenheimer Feld 288, 69120 Heidelberg, Germany (bouganis@mathi.uni-heidelberg.de).

${ }^{\ddagger}$ Universität Heidelberg, Mathematisches Institut, Im Neuenheimer Feld 288, 69120 Heidelberg, Germany (venjakob@mathi.uni-heidelberg.de).
} 
and

$$
S^{*}=\bigcup_{n \geq 0} p^{n} S
$$

The following theorem compromises the technical heart of [7].

THEOREM 1.1. The sets $S$ and $S^{*}$ are (left and right) Ore sets, i.e. the localisations $\Lambda_{S}$ and $\Lambda_{S^{*}}$ of $\Lambda$ exist and the following holds:

(i) The category of all finitely generated $S^{*}$-torsion $\Lambda(\mathcal{G})$-modules coincides with $\mathfrak{M}_{\mathcal{H}}(\mathcal{G})$.

(ii) There is an long exact localisation sequence of $K$-groups

$$
K_{1}(\Lambda(\mathcal{G})) \longrightarrow K_{1}\left(\Lambda(\mathcal{G})_{S^{*}}\right) \stackrel{\partial}{\longrightarrow} K_{0}\left(\mathfrak{M}_{\mathcal{H}}(\mathcal{G}) \longrightarrow 0\right.
$$

and analogously for $\Lambda(\mathcal{G})_{S}$ and the category of finitely generated $S$-torsion modules.

(iii) There is a canonical way of evaluation an element $f \in K_{1}\left(\Lambda(\mathcal{G})_{S^{*}}\right)$ at any continuous representation $\rho: \mathcal{G} \rightarrow G L_{n}(\mathcal{O})$ with $n \geq 1$ and $\mathcal{O}$ the ring of integers of a finite extension (depending of $\rho$ ) of $\mathbb{Q}_{p}$ :

$$
f(\rho) \in \mathbb{C}_{p} \cup\{\infty\},
$$

i.e. $f$ can be considered as a map on the set of such representations.

Proof. See [31, 7]. Another proof that $S$ is an Ore-set was given by Ardakov and Brown in [1]. The pseudo-compact case (generalised to certain skew power series rings) is covered by [23] or can be proven in the same way as in [7]. $\square$

By $S\left(E / F_{\infty}\right)$ and $X=X\left(E / F_{\infty}\right)=S\left(E / F_{\infty}\right)^{\vee}$ we denote the (classical) $p$ primary Selmer group of $E$ over $F_{\infty}$ and its Pontryagin dual, respectively. It is easy to see that $X$ is a finitely generated $\Lambda_{\mathbb{Z}_{p}}(\mathcal{G})$-module with the natural Galois-action on $S\left(E / F_{\infty}\right)$, but the following torsion-property can be interpreted as a generalization of a deep conjecture of Mazur.

Conjecture 1.2 (Torsion-conjecture). The dual of the Selmer group is $S^{*}$ torsion:

$$
X\left(E / F_{\infty}\right) \in \mathfrak{M}_{\mathcal{H}}(\mathcal{G})
$$

Now let $K\left(F_{\infty}\right)$ be the maximal abelian extension of $\mathbb{Q}$ inside $F_{\infty}$ in which $p$ does not ramify and $L=K\left(F_{\infty}\right)_{\mathfrak{P}}$ its completion at some $\mathfrak{P}$ lying over $p$. Note that $L$ is a finite extension of $\mathbb{Q}_{p}$. Finally we put $D=\mathcal{O}_{L}$.

If $\omega$ denotes the Neron differential of $E$, we obtain the usual real and complex periods $\Omega_{ \pm}=\int_{\gamma^{ \pm}} \omega$ by integrating along pathes $\gamma^{ \pm}$which generate $H_{1}(E(\mathbb{C}), \mathbb{Z})^{ \pm}$. We set $R=\left\{q\right.$ prime, $\left.|j(E)|_{q}>1\right\} \cup\{p\}$ and let $u, w$ be the roots of the characteristic polynomial of the action of Frobenius on the Tate-module $T_{p} E$ of $E$

$$
1-a_{p} T+p T^{2}=(1-u T)(1-w T), \quad u \in \mathbb{Z}_{p}^{\times} .
$$


Further let $p^{f_{p}(\rho)}$ be the $p$-part of the conductor of an Artin representation $\rho$, while $P_{p}(\rho, T)=\operatorname{det}\left(1-\right.$ Frob $\left._{p}^{-1} T \mid V_{\rho}^{I_{p}}\right)$ describes the Euler-factor of $\rho$ at $p$. We also set $d^{ \pm}(\rho)=\operatorname{dim}_{\mathbb{C}} V_{\rho}^{ \pm}$and denote by $\stackrel{\vee}{\rho}$ the contragredient representation of $\rho$. By $e_{p}(\rho)$ we denote the local $\epsilon$-factor of $\rho$ at $p$. In the notation of [29] this is $e_{p}\left(\rho, \psi(-x), d x_{1}\right)$ where $\psi$ is the additive character of $\mathbb{Q}_{p}$ defined by $x \rightarrow \exp (2 \pi i x)$ and $d x_{1}$ is the Haar measure that gives volume 1 to $\mathbb{Z}_{p}$. Finally, in order to express special values of complex $L$-functions in the $p$-adic world, we fix embeddings of $\overline{\mathbb{Q}}$ into $\mathbb{C}$ and $\mathbb{C}_{p}$, the completion of an algebraic closure of $\mathbb{Q}_{p}$.

Conjecture 1.3 (Existence of $p$-adic $L$-function). There is a (unique) $\mathcal{L}_{E} \in$ $K_{1}\left(\Lambda(\mathcal{G})_{S^{*}}\right)$ such that

$$
\mathcal{L}_{E}(\stackrel{\vee}{\rho})=\frac{L_{R}(E, \rho, 1)}{\Omega_{+}^{d^{+}(\rho)} \Omega_{-}^{d^{-}(\rho)}} e_{p}(\check{\rho}) \frac{P_{p}\left(\stackrel{\vee}{\rho}, u^{-1}\right)}{P_{p}\left(\rho, w^{-1}\right)} u^{-\mathfrak{f}_{p}(\check{\rho})}
$$

for all Artin representations $\rho$ of $\mathcal{G}$.

For a class $[M] \in K_{0}\left(\mathfrak{M}_{\mathcal{H}}(\mathcal{G})\right)$ we denote by $[M]_{D}$ the base change to $K_{0}\left(\mathfrak{M}_{D, \mathcal{H}}(\mathcal{G})\right)$.

Conjecture 1.4 (Main Conjecture). The p-adic L-function $\mathcal{L}_{E}$ is a characteristic element of $X\left(E / F_{\infty}\right)$ :

$$
\partial \mathcal{L}_{E}=\left[X\left(E / F_{\infty}\right)\right]_{D}
$$

We refer the reader to $[5,6]$ where a refined version involving leading terms is discussed and where some implications of the Main Conjecture are explained.

2. The CM-case. In this section we assume that $E$ is still defined over $\mathbb{Q}$, has conductor $N:=N_{E}$ and moreover admits complex multiplication by the ring of integers $\mathcal{O}_{K}$ of a quadratic imaginary field $K=\mathbb{Q}\left(\sqrt{-d_{K}}\right)$, where cf. Appendix A $\S 3$ of [27] the discriminant $d_{K}>0$ lies in the finite set

$$
\{3,4,7,8,11,19,43,67,163\} \text {. }
$$

Note that since $j(E) \in \mathbb{Q}$ the class number of $K$ is trivial: $h_{K}=1$. As we assume that $E$ has good ordinary reduction at our fixed prime $p$, the latter decomposes into two primes $\mathfrak{p}=(\pi)$ and $\overline{\mathfrak{p}}=(\bar{\pi})$, such that $\pi$ and $\bar{\pi}$ are conjugate under $c \in \Delta:=$ $G(K / \mathbb{Q})$ which is induced by complex conjugation. We fix an embedding of $K$ into $\overline{\mathbb{Q}}$ such that $\mathfrak{p}$ corresponds to the induced embedding of $K$ into $\mathbb{C}_{p}$ (using the convention of the previous section).

Setting $H:=G\left(K_{\infty} / K_{c y c}\right), G:=\left(K_{\infty} / K\right)$ we obtain isomorphisms $G / H \cong \Gamma$ and $\mathcal{H} / H \cong \Delta \cong \mathcal{G} / G$. The situation can be illustrated by the following diagram of field extensions: 


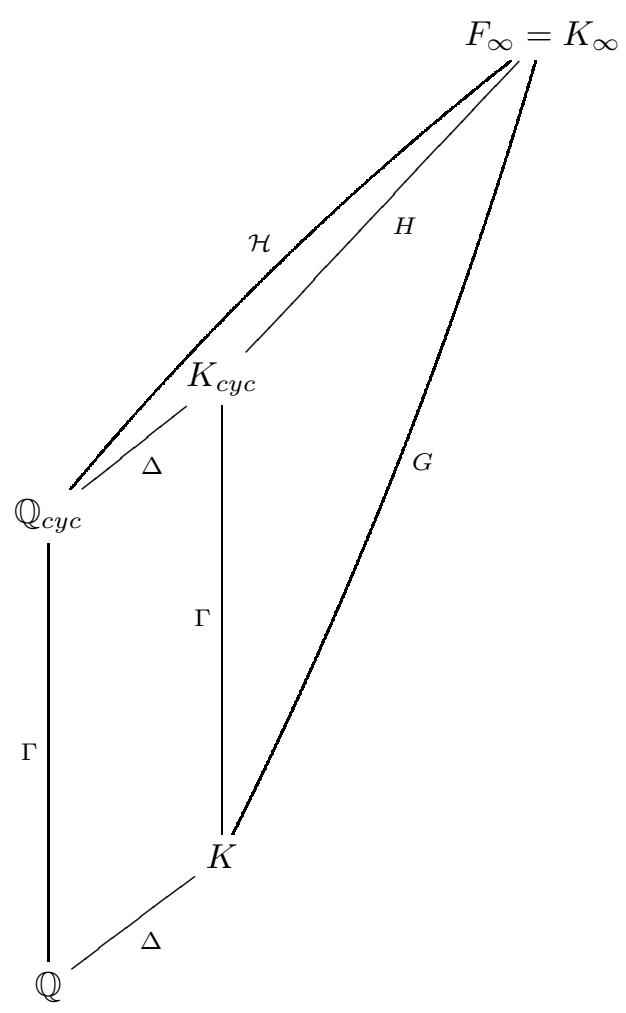

In particular the non-commutative group $\mathcal{G}$ is an extension of the cyclic group of order 2 by the abelian group $G$, i.e. very close to being abelian.

One strategy to verify the Main Conjecture for $E$ in the previous section would be a close analysis how the groups $K_{1}\left(\Lambda(\mathcal{G})_{S^{*}}\right)$ and $K_{0}\left(\mathfrak{M}_{\mathcal{H}}(\mathcal{G})\right)$ look like, in order to construct the (non-commutative) $p$-adic $L$-function and to know how to show that two classes in $K_{0}\left(\mathfrak{M}_{\mathcal{H}}(\mathcal{G})\right)$ in the absence of a suitable structure theory. This certainly can be done, but we will follow a much simpler way.

In this work we are going to make the following assumption:

Assumption. The Conjecture 1.2 in the CM case is true.

REMARK 2.1. We remark that this assumption implies that $X\left(E / F_{\infty}\right)$ is actually $S$-torsion. For this one has simply to show that the $\mu$-invariant of $X\left(E / F_{\infty}\right)$ is trivial since it is known by [18, théorème 2.4$]$ that $X\left(E / F_{\infty}\right)$ contains no non-trivial pseudonull $\Lambda(G)$ submodules. But the triviality of the $\mu$-invariant follows from the results of Schneps [24] after employing a descent argument similar to the one done in $[14$, theorem 5.3].

The key ingredient for our proof of the Main Conjecture is the fact that to $E$ there is attached a $A_{0^{-}}$Größencharacter, in the sense of Weil, $\psi$ of weight $(1,0)$ and conductor $\mathfrak{f}=\mathfrak{f}_{\psi}$ with $N=d_{K} N_{K / \mathbb{Q}}(\mathfrak{f})$ such that

$$
" E=\operatorname{Ind}_{\mathbb{Q}}^{K} \psi ",
$$

This can either be understood 
(i) in the sense of the attached compatible system of $l$-adic Galois representations. Indeed to the character $\psi$ there is by Weil attached a compatible system of $\ell$-adic representations and then the corresponding system of $\ell$-adic representations of $E$ are obtained by induction from the absolute Galois group of $K$ to the absolute Galois group of $\mathbb{Q}$.

(ii) or in the automorphic induction sense. Indeed the automorphic counterpart of $\psi$ is an adelic character $\psi: \mathbb{A}_{K}^{\times} / K^{\times} \rightarrow \mathbb{C}^{\times}$and the corresponding cuspidal automorphic representation $\pi_{E}$ of $G L_{2}\left(\mathbb{A}_{\mathbb{Q}}\right)$ associated to $E$ is obtained by automorphic induction from $G L_{1}\left(\mathbb{A}_{K}\right)$ to $G L_{2}\left(\mathbb{A}_{\mathbb{Q}}\right)$.

There is also a Größencharacter $\bar{\psi}$ of weight $(0,1)$ (attached to $E$ ). As it was first shown by Weil these characters although being adelic in nature can also be interpreted as Galois characters of $G\left(K\left(\mathfrak{f} p^{\infty}\right) / K\right)$ or $G\left(K_{\infty} / K\right)$ with values in $\mathbb{Z}_{p}^{*}$, see also [9, page 38]. Note that, since $E$ is defined over $\mathbb{Q}$, it holds by [17, page 559] that

$$
\bar{\psi}=\psi^{c}
$$

where $\psi^{c}(g)=\psi\left(c g c^{-1}\right)$ for all $g \in G\left(K_{\infty} / K\right)$.

By the functorial properties of $K$ - and representation theory and using (2.1) the desired result can be reduced to the two-variable Main Conjecture as proved by Rubin. In order to state it we introduce $\mathcal{X}\left(K_{\infty}\right)$ to be the Galois group of the maximal abelian $p$-extension of $K_{\infty}$ which is unramified outside $\mathfrak{p}$. This is a finitely generated torsion $\Lambda(G)$-module, [21, page 37]. Furthermore, let

$$
T_{\pi}:=T_{\pi} E:=\lim _{n} E\left[\pi^{n}\right]
$$

be the $\pi$-primary Tate-module of $E$, similarly for $\bar{\pi}$, and

$$
T_{\pi}^{*}=\operatorname{Hom}\left(T_{\pi}, \mathbb{Z}_{p}\right)
$$

its $\mathbb{Z}_{p}$-dual representation of $G_{K}$. Note that the action of $G_{K}$ on $T_{\pi}$ and $T_{\bar{\pi}}$ is given by the characters $\psi$ and $\bar{\psi}$, respectively. Our above philosophy is confirmed by the following

Proposition 2.2. There is a natural isomorphism of $\Lambda(\mathcal{G})$-modules

$$
X\left(E / K_{\infty}\right) \cong \operatorname{Ind}_{\mathcal{G}}^{G}\left(\mathcal{X}\left(K_{\infty}\right) \otimes_{\mathbb{Z}_{p}} T_{\pi}^{*}\right),
$$

where $G$ acts diagonally on the tensor product $\mathcal{X}\left(K_{\infty}\right) \otimes_{\mathbb{Z}_{p}} T_{\pi}^{*} \cong \mathcal{X}\left(K_{\infty}\right)\left(\psi^{-1}\right)$.

The proof is a modification of the proof of the old observation by John Coates that the $\pi$-Selmer group $S_{\pi}\left(E / K_{\infty}\right)$ of $E$ over $K_{\infty}$ is canonically isomorphic to

$$
S_{\pi}\left(E / K_{\infty}\right) \cong \operatorname{Hom}\left(\mathcal{X}\left(K_{\infty}\right), E(\pi)\right) .
$$

Proof. Conferring to $[9$, page 124$]$ the $\pi$-primary Selmer group is given by the following exact sequence

$$
0 \longrightarrow S_{\pi}\left(E / K_{\infty}\right) \longrightarrow \mathrm{H}^{1}\left(G_{S_{p}}\left(K_{\infty}\right), E(\pi)\right) \longrightarrow \operatorname{Coind}_{G}^{G_{\bar{p}}} \mathrm{H}^{1}\left(K_{\infty, \nu}, E\right)(\pi)
$$

where the coinduction is dual to the induction functor, $\nu$ denotes a prime of $K_{\infty}$ above $\overline{\mathfrak{p}}$. Applying the exact functor $\operatorname{Coind}_{\mathcal{G}}^{G}$, using the transitivity of coinduction, the isomorphisms

$$
\operatorname{Coind}_{\mathcal{G}}^{G} \mathrm{H}^{1}\left(G_{S_{p}}\left(K_{\infty}\right), E(\pi)\right) \cong \operatorname{Hom}\left(G_{S_{p}}\left(K_{\infty}\right), \operatorname{Coind}_{\mathcal{G}}^{G} E(\pi)\right) \cong H^{1}\left(G_{S_{p}}\left(K_{\infty}\right), E(p)\right)
$$


and

$$
\mathrm{H}^{1}\left(K_{\infty, \nu}, E\right)(p) \cong \mathrm{H}^{1}\left(K_{\infty, \nu}, E\right)(\pi) \oplus \mathrm{H}^{1}\left(K_{\infty, \nu}, E\right)(\bar{\pi}),
$$

as well as the vanishing (see loc. cit.) of $\mathrm{H}^{1}\left(K_{\infty, \nu}, E\right)(\bar{\pi})$, one just obtains the defining sequence of the full $p$-primary Selmer group

$$
0 \longrightarrow S\left(E / K_{\infty}\right) \longrightarrow \mathrm{H}^{1}\left(G_{S_{p}}\left(K_{\infty}\right), E(p)\right) \longrightarrow \operatorname{Coind}_{\mathcal{G}}^{\mathcal{G}_{\bar{F}}} \mathrm{H}^{1}\left(K_{\infty, \nu}, E\right)(p) .
$$

The result now follows by taking duals.

In order to introduce the (commutative) two-variable $p$-adic $L$-function we choose a complex period $\Omega_{\infty} \in \mathbb{C}^{\times}$such that $\Lambda_{E}=\Omega_{\infty} \mathfrak{f}_{\psi}$ for the period lattice $\Lambda_{E}$ attached to the pair $(E, \omega)$. Here we view $\mathfrak{f}_{\psi}$ as a lattice in $\mathbb{C}$ with respect the chosen embedding $K \hookrightarrow \mathbb{C}$ that correspond to the type of $\psi$. This determines the $p$-adic period $\Omega_{p} \in$ $\widehat{\mathbb{Z}}_{p}^{n r} \times$ by the procedure described in $[9$, page 66$]$ such that the ratio $\frac{\Omega_{\infty}}{\Omega_{p}}$ is independent of any choices. Actually we can pin down the $p$-adic $\Omega_{p}$ up to elements in $\mathbb{Z}_{p}^{\times}$by the rule $\frac{\Omega_{p}^{\Phi}}{\Omega_{p}}=u$ where $\Phi$ is the extension of Frobenious to $\widehat{\mathbb{Z}_{p}^{n r}}$. Then we have:

Theorem 2.3 (Manin-Višik, Katz, Yager, de Shalit). Let $\mathfrak{f}$ be an integral ideal of $K$ relative prime to $p$. For $D=\widehat{\mathbb{Z}_{p}^{n r}}$ there is a unique measure $\mu\left(\mathfrak{f p}^{\infty}\right) \in$ $\Lambda_{D}\left(G\left(K\left(\mathfrak{f} p^{\infty}\right) / K\right)\right)$ such that for any grössencharacter $\epsilon$ of conductor dividing $\mathfrak{f} p^{\infty}$ and of type $(k, j)$ with $0 \leq-j$ and $k>0$ we have,

$$
\Omega_{p}^{j-k} \int_{G\left(K\left(\mathfrak{f} p^{\infty}\right) / K\right)} \epsilon^{-1}(\sigma) \mu\left(\mathfrak{f} \overline{\mathfrak{p}}^{\infty}\right)=\Omega_{\infty}^{j-k} \Gamma(k) i^{k}\left(\frac{\sqrt{d_{K}}}{2 \pi}\right)^{j} G(\epsilon)\left(1-\frac{\epsilon(\mathfrak{p})}{p}\right) L_{\overline{\mathfrak{p}} \mathfrak{f}}\left(\epsilon^{-1}, 0\right)
$$

where

$$
G(\epsilon):=\frac{\phi^{k} \bar{\phi}^{j}\left(\mathfrak{p}^{n}\right)}{p^{n}} G(\chi):=\frac{\phi^{k} \bar{\phi}^{j}\left(\mathfrak{p}^{n}\right)}{p^{n}} \sum_{\gamma \in M} \chi(\gamma)\left(\zeta_{n}^{\gamma}\right)^{-1}
$$

Here we write $\epsilon=\phi^{k} \bar{\phi}^{j} \chi$ with $\phi$ a Grössencharacter of conductor prime to $\mathfrak{p}$ and of type $(1,0)$ and $\chi$ a character of finite order of conductor at $\mathfrak{p}$ equal to $\mathfrak{p}^{n}$ and

$$
M:=\left\{\gamma \in \operatorname{Gal}\left(K\left(\mathfrak{f} \overline{\mathfrak{p}}^{\infty} \mathfrak{p}^{n}\right) / K\right) \mid \gamma_{\mid F^{\prime}}=\left(\mathfrak{p}^{n}, F^{\prime} / K\right)\right\}, \quad F^{\prime}:=K\left(\mathfrak{f} \overline{\mathfrak{p}}^{\infty}\right) .
$$

Proof. This theorem is from [9, thm. II 4.14] (where the restriction on the type of the character is that $0 \leq-j<k$ ) in combination with corollary 6.7 of the same book. We note here that the relation between the Galois counterpart of $\epsilon$ that appear in the left hand side of the above formula and its automorphic counterpart that shows up in the right side are related by $\epsilon^{\text {Galois }}\left(\right.$ Frob $\left._{\mathfrak{q}}^{-1}\right)=\epsilon^{\text {aut }}(\mathfrak{q})$, is different from deShalit who sets $\epsilon^{\text {Galois }}\left(\right.$ Frob $\left._{\mathfrak{q}}\right)=\epsilon^{\text {aut }}(\mathfrak{q})$. This explains the difference of the formula above with the one of deShalit in page 80 (the argument of the integral is inverted). We also need to remark that in the theorem of de Shalit also the $\Gamma$-factor of the $L$-function appears which is equal to $(2 \pi)^{-1}$. However in de Shalit the archimedean period used is equal to $(2 \pi)^{-1} \Omega_{\infty}$ (compare theorem 4.11 and 4.12 in [9]). Finally the factor $i^{k}$ is coming from the normalization of the $p$-adic periods (see [9, page 70$]$ ).

For our purposes it is important to understand the relation of the "Gauss sum" like factor with the epsilon factor at $\mathfrak{p}$. To this end we have the following lemma, 
Lemma 2.4. Let $\delta \in \mathbb{Z}_{p}{ }^{\times}$be the image of $\sqrt{-d_{K}}$ under $K \hookrightarrow K_{\mathfrak{p}}=\mathbb{Q}_{p}$ and $\sigma_{\delta} \in \operatorname{Gal}\left(K\left(\mathfrak{f} p^{\infty}\right) / K\left(\mathfrak{f} \overline{\mathfrak{p}}^{\infty}\right)\right)$ such that $\sigma_{\delta}(\zeta)=\zeta^{\delta}$ for all $p$-power roots of unity. Then with notation as in the theorem above we have

$$
G(\chi)=\chi\left(\sigma_{\delta}\right) e_{\mathfrak{p}}(\chi)
$$

Proof. We base the following proof on the article of Tate [29] and [9] section 6.3. By [9] (page 92) we have

$$
e_{\mathfrak{p}}\left(\epsilon, \psi, d x_{1}\right)^{-1}=p^{\frac{n}{2}(k+j+1)} \delta^{-k} \epsilon\left(\sigma_{\delta}\right) G\left(\epsilon^{-1}\right) .
$$

From the definition of $\delta \in \mathbb{Z}_{p}{ }^{\times}$we have $\bar{\phi}\left(\sigma_{\delta}\right)=1$ and $\phi\left(\sigma_{\delta}\right)=\delta$ (see also [9] (page 92). In the above equation we set $\epsilon=\bar{\chi} \phi^{-1}$ where $\phi$ as always a Grössencharacter of type $(1,0)$. In particular we have that $\epsilon$ is a character of type $(-1,0)$. The above equation reads (with $k=-1$ and $j=0$ )

$$
e_{\mathfrak{p}}\left(\bar{\chi} \phi^{-1}, \psi(x), d x_{1}\right)^{-1}=\delta \bar{\chi}\left(\sigma_{\delta}\right) \phi^{-1}\left(\sigma_{\delta}\right) G(\chi \phi) .
$$

Now we note that $\phi^{-1}\left(\sigma_{\delta}\right)=\delta^{-1}$ and since $\phi$ is unramified at $\mathfrak{p}$ we have that (see [29]) $e_{\mathfrak{p}}\left(\phi^{-1} \bar{\chi}, \psi(x), d x_{1}\right)=\phi(\mathfrak{p})^{-n} e_{\mathfrak{p}}\left(\bar{\chi}, \psi(x), d x_{1}\right)$. With these remarks the above equation simplifies to

$$
e_{\mathfrak{p}}\left(\bar{\chi}, \psi(x), d x_{1}\right)^{-1} \phi(\mathfrak{p})^{n}=\bar{\chi}\left(\sigma_{\delta}\right) G(\chi \phi) .
$$

Using now the duality property (see [29])

$$
e_{\mathfrak{p}}\left(\bar{\chi}, \psi(x), d x_{1}\right) e_{\mathfrak{p}}\left(\chi, \psi(-x), d x_{1}\right)=p^{n}
$$

we obtain that

$$
e_{\mathfrak{p}}\left(\chi, \psi(-x), d x_{1}\right) p^{-n} \phi(\mathfrak{p})^{n}=\bar{\chi}\left(\sigma_{\delta}\right) G(\chi \phi) .
$$

But $G(\chi \phi)=\frac{\phi(\mathfrak{p})^{n}}{p^{n}} G(\chi)$ from which we conclude that

$$
G(\chi)=\chi\left(\sigma_{\delta}\right) e_{\mathfrak{p}}\left(\chi, \psi(-x), d x_{1}\right)=\chi\left(\sigma_{\delta}\right) e_{\mathfrak{p}}(\chi)
$$

following our convention to write $e_{\mathfrak{p}}(\cdot)$ for $e_{\mathfrak{p}}\left(\cdot, \psi(-x), d x_{1}\right)$.

Corollary 2.5. There is a unique $\mathcal{L}_{\bar{\psi}}^{\prime}:=\mu \in \Lambda_{D}(G)$ such that

$$
\mathcal{L}_{\bar{\psi}}^{\prime}(\bar{\chi})=\int_{G} \chi d \mu=\frac{L(\bar{\psi} \chi, 1)}{\Omega_{\infty}} e_{\mathfrak{p}}(\bar{\chi}) P_{\mathfrak{p}}\left(\bar{\chi}, u^{-1}\right) P_{\bar{p}}\left(\chi, u^{-1}\right) u^{-\mathfrak{f}_{\mathfrak{p}}(\bar{\chi})}
$$

for all Artin-character $\chi$ of $G$.

Proof. This is in principle the measure mentioned in the above theorem 2.3 twisted by the fixed character $\psi$. However in the theorem above it is the imprimitive $L$ function that appears (as we remove the Euler factors at $\mathfrak{f}$ even if the conductor of the character is not $\mathfrak{f}$ ). For our purposes we need the primitive $L$-functions. We now explain how it can be constructed. We consider the decomposition $\operatorname{Gal}\left(K_{\infty} / K\right)=$ $G \cong \Delta \times G_{1}$ where $\Delta \cong(\mathbb{Z} / p \mathbb{Z})^{\times} \times(\mathbb{Z} / p \mathbb{Z})^{\times}$and $G_{1} \cong \mathbb{Z}_{p}^{2}$. This decomposition induces a decomposition of Iwasawa algebras

$$
\Lambda_{D}[[G]]=D[[G]] \cong D[\Delta]\left[\left[G_{1}\right]\right] \cong \oplus_{\theta \in \hat{\Delta}} D\left[\left[G_{1}\right]\right]
$$


where $\hat{\Delta}=\operatorname{Hom}\left(\Delta, D^{\times}\right)$the dual group of $\Delta$. For a character $\chi$ of $G$ we consider its decomposition into $\chi=\chi_{\Delta} \chi_{1}$ according to the above decomposition of $G$. Similarly we have $\psi=\psi_{\Delta} \psi_{1}$. We are going to define the element $\mu \in \Lambda_{D}[[G]]$ claimed in the proposition by defining the elements in $D\left[\left[G_{1}\right]\right]$ in the above decomposition of the Iwasawa algebras. We define the $\theta^{\text {th }}$ component $\mu_{\theta}$ as follows. We write $\mathfrak{g}$ for the prime to $p$ part of the conductor of the character $\theta \psi_{\Delta}$. We note that $\mathfrak{g}$ can be different from $\mathfrak{f}_{\psi}$. We consider the measure $\delta \sigma_{\delta}^{-1} \star \Omega_{p}^{-1} \mu\left(\mathfrak{g} \overline{\mathfrak{p}}^{\infty}\right) \in \Lambda_{D}\left(G\left(K\left(\mathfrak{g} p^{\infty}\right) / K\right)\right.$ ) (here $\star$ denotes convolution of measures) and define the measure $\mu_{\theta}$ on $G_{1}$ as

$$
\int_{G_{1}} f d \mu_{\theta}=\frac{1}{\Omega_{p}} \int_{G\left(K\left(\mathfrak{g} p^{\infty}\right) / K\right)} \psi^{-1} \theta \cdot(f \circ p r) d\left(\delta \sigma_{\delta}^{-1} \star \mu\left(\mathfrak{g} \overline{\mathfrak{p}}^{\infty}\right)\right)
$$

for all integrable functions $f$ on $G_{1}$. Here $p r: G\left(K\left(\mathfrak{g} p^{\infty}\right) / K\right) \rightarrow G_{1}$ denotes the natural projection map and $\psi \theta$ is seen as a character of $G\left(K\left(\mathfrak{g} p^{\infty}\right) / K\right)$. We define the measure $\mu$ by putting together the components $\mu_{\theta}$ according to the isomorphism $\Lambda_{D}[[G]] \cong \oplus_{\theta \in \hat{\Delta}} D\left[\left[G_{1}\right]\right]$. Now the result follows from the above theorem by taking $k=1, j=0, \epsilon=\psi \bar{\chi}$ and noting that $\mathfrak{f}_{\mathfrak{p}}(\bar{\chi} \psi)=\mathfrak{f}_{\mathfrak{p}}(\bar{\chi})=\mathfrak{f}_{\mathfrak{p}}(\chi)$ because $\psi$ is unramified at $\mathfrak{p}$. Also note that from the equation

$$
(1-u X)(1-w X)=1-a_{p} X+p X^{2}=(1-\psi(\mathfrak{p}) X)(1-\psi(\overline{\mathfrak{p}}) X)
$$

and the condition that $u$ is a unit in $\mathbb{Z}_{p}$, it follows that

$$
u=\psi(\overline{\mathfrak{p}})=\bar{\pi}
$$

and

$$
u^{-1}=\frac{w}{p}=\frac{\psi(\mathfrak{p})}{p}
$$

COROllary 2.6. There is a unique $\mathcal{L}_{\bar{\psi}}:=\mu \in \Lambda_{D}(G)$ such that

$$
\mathcal{L}_{\bar{\psi}}(\bar{\chi})=\int_{G} \bar{\chi} d \mu=\frac{L(\bar{\psi} \chi, 1)}{\Omega_{\infty}} e_{\overline{\mathfrak{p}}}(\bar{\chi}) P_{\mathfrak{p}}\left(\bar{\chi}, u^{-1}\right) P_{\overline{\mathfrak{p}}}\left(\chi, u^{-1}\right) u^{-\mathfrak{f}_{\bar{p}}(\bar{\chi})}
$$

for all Artin-character $\chi$ of $G$.

Proof. This measure is just a twist of the measure $\mathcal{L}_{\bar{\psi}}^{\prime}$ by a unit in $\Lambda_{D}(G)$. This follows from the functional equation of the $L$-function involved. We defer the proof of this corollary to the next section (compare with corollary 3.6 and corollary 3.7 ).

Now we are ready to state the two-variable Main Conjecture.

Theorem 2.7 (Rubin,Yager). As ideals in $\Lambda_{D}(G)$ there is the following equality

$$
\operatorname{char}\left(\mathcal{X}\left(K_{\infty}\right) \otimes_{\mathbb{Z}_{p}} T_{\pi}^{*}\right)=\left(\mathcal{L}_{\bar{\psi}}\right),
$$

where the left hand side denotes the characteristic ideal associated with the $\Lambda(G)$ torsion module $\mathcal{X}\left(K_{\infty}\right) \otimes_{\mathbb{Z}_{p}} T_{\pi}^{*}$ by the structure theory from commutative algebra.

REMARK 2.8. With respect to the two-variable Main Conjecture stated above we would like to make some comments. In principle the theorem above follows by combining [21, page 37, Theorem 4.1] of the work of Rubin with [32, page 413, Theorem 
1] of Yager. However there is a subtle issue with respect to the $p$-adic $L$-function. The one constructed by Yager in (loc. cit.) and stated as theorem 2.3 above does not interpolate the primitive $L$-function (see also the comment of Yager in pages 413414). On the other hand there is a remark by Rubin (remark (1) in page 37 in (loc. cit.)) which we believe deserves some more explanation. There Rubin writes that the $p$-adic $L$-function of Yager is the characteristic element of $U_{\infty} / \bar{C}_{\infty}$ (local units modulo completion of elliptic units) as defined in his work. However the elliptic units in Rubin's work do not coincide with those considered by Yager. Indeed, the ones used by Rubin are the ones that satisfy the analytic class number formula [21, Theorem 1.3] for abelian extensions $F$ of $K$ and these are defined for example as in the work of Gillard [12, section 6] or Gillard and Robert [13, page 309]. The main point is that one should consider carefully the various subfields of $F$ and their conductors. On the other hand the Grössencharacter $\psi^{k}$ may have conductor different from $\mathfrak{f}$ for the various $k$ 's. As it is shown in the work of Bernardi, Goldstein and Stephens [2] when one takes care also of these considerations (i.e. working carefully the various eigenspaces of the torsion part of $G$ ) then one obtains the $p$-adic $L$-function that interpolates primitive $L$-values through the usual machinery of logarithmic derivatives. ${ }^{1}$ Indeed, the logarithmic derivatives of the elliptic functions considered in the latter work give the primitive $L$-values and these elliptic functions are slightly different from the ones in Yager's work. The elliptic units in Rubin's work are obtained as values of elliptic functions similar to the ones used in the work of Bernardi, Goldstein and Stephens, which implies that the characteristic element of $U_{\infty} / \bar{C}_{\infty}$ in Rubin's work is indeed the $p$-adic $L$-function that interpolates primitive values.

Lemma 2.9. The module $\mathcal{X}\left(K_{\infty}\right) \otimes_{\mathbb{Z}_{p}} T_{\pi}^{*}$ is $S$-torsion, in particular $\mathcal{X}\left(K_{\infty}\right) \otimes_{\mathbb{Z}_{p}}$ $T_{\pi}^{*} \in \mathfrak{M}_{H}(G)$, and thus $\mathcal{L}_{\bar{\psi}} \in\left(\Lambda(G)_{S}\right)^{\times} \subseteq\left(\Lambda(G)_{S^{*}}\right)^{\times}$.

Proof. By Proposition $2.2 \mathcal{X}\left(K_{\infty}\right) \otimes_{\mathbb{Z}_{p}} T_{\pi}^{*}$ being $S$-torsion is equivalent to $X\left(E / K_{\infty}\right)$ being $S$-torsion, which is just remark 2.1. The rest of the claim follows from the two-variable Main Conjecture above and the commutative diagram

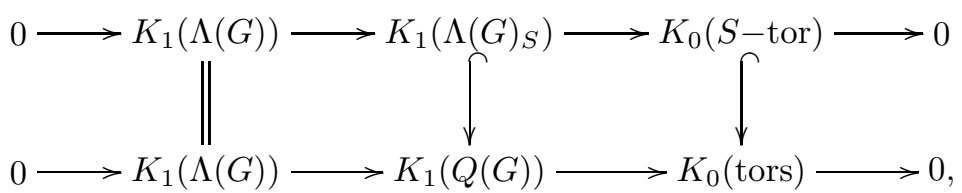

where $Q(G)$ denotes the maximal ring of quotients of $\Lambda(G)$, while tors denotes the category of all finitely generated $\Lambda(G)$-torsion modules.

Note that, although the Ore-sets $S_{G}^{*}$ and $S_{\mathcal{G}}^{*}$ are different in general, we have a natural isomorphism $\Lambda(\mathcal{G})_{S_{\mathcal{G}}^{*}} \cong \Lambda(\mathcal{G})_{S_{G}^{*}}$ by [23, Lemma 4.6] where in the second case the localization is formed with respect to the $G$-module structure of $\Lambda(\mathcal{G})$. Hence we have a commutative base change square

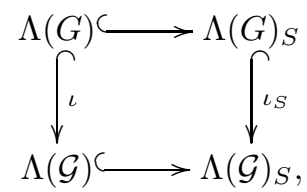

\footnotetext{
${ }^{1}$ In their work they consider the one variable $p$-adic $L$-function but one can extend their considerations to obtain the primitive two variable $p$-adic $L$-function applying the machinery developed by Yager.
} 
which induces the following diagram with exact rows by functoriality of $K$-theory

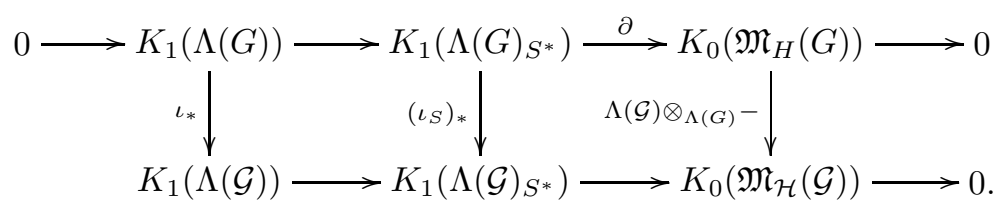

It follows that $\mathcal{L}:=\left(\iota_{S}\right)_{*}\left(\mathcal{L}_{\bar{\psi}}\right)$ is automatically a characteristic element for $\left[X\left(E / K_{\infty}\right)\right]$ by Proposition 2.2. A naive hope would be that this is the desired $p$-adic $L$-function for $E$. In order to check this we need the next lemma which describes the evaluation of $\mathcal{L}$ at representations.

Lemma 2.10. For all Artin representations $\rho$ of $\mathcal{G}$ one has

$$
\mathcal{L}(\rho)=\mathcal{L}_{\bar{\psi}}\left(\operatorname{Res}_{G}^{\mathcal{G}} \rho\right),
$$

where $\operatorname{Res}_{G}^{\mathcal{G}} \rho$ denotes the restriction to the subgroup $G$. With other words $\mathcal{L}$ is induced by the measure $\mu_{\mathcal{L}_{\bar{\psi}}}$ trivially extended

$$
\int_{\mathcal{G}} f d \mu_{\mathcal{L}}=\int_{G} f_{\mid G} d \mu_{\mathcal{L}_{\bar{\psi}}},
$$

i.e. from the image of $\mu_{\mathcal{L}_{\bar{\psi}}}$ under the natural map $\Lambda(G) \hookrightarrow \Lambda(\mathcal{G})$.

Proof. Upon comparing with the definition of evaluation in [7] the statement follows from the following diagram, which is obviously commutative:

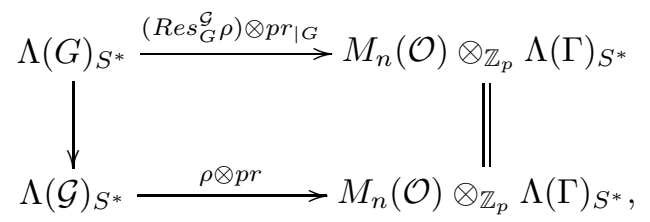

where $p r: \Lambda(\mathcal{G})_{S^{*}} \rightarrow \Lambda(\Gamma)_{S^{*}}$ denotes the canonical projection.

The next task is to understand the (irreducible) Artin representations of $\mathcal{G}$. By $[25$, page 62$]$ and the fact that $\mathcal{G}$ is a semi-direct product

$$
\mathcal{G} \cong G \rtimes \Delta
$$

one immediately obtains that an irreducible such representation is either of

Typ A $: \rho$ is one dimensional,

or

Typ B: $\rho=\operatorname{Ind}_{\mathcal{G}}^{G} \chi$ is of dimension two where $\chi$ is a one-dimensional character of $G$ with $\chi \neq \chi^{c}$.

Indeed to see that the only irreducible representations of dimension bigger than one are those of dimension two and they are of the form described above one has simply to use Frobenious reciprocity. For, if an Artin representation $\rho$ of $\mathcal{G}$ has dimension $n$ with $n \geq 2$ and we consider its restriction to $G$ then $\operatorname{Res}_{\mathcal{G}}^{G} \rho=\oplus_{i=1}^{n} \chi_{i}$ for $n$ one dimensional representations of $G$. But then for every $\chi_{j}$, with $1 \leq j \leq n$, by applying Frobenious Reciprocity we have

$$
0 \neq\left(\operatorname{Res}_{\mathcal{G}}^{G} \rho, \chi_{j}\right)_{G}=\left(\rho, \operatorname{Ind} d_{\mathcal{G}}^{G} \chi_{j}\right)_{\mathcal{G}}
$$


and the representation $\operatorname{Ind} d_{\mathcal{G}}^{G} \chi_{j}$ is of dimension two. That is, every representation of dimension greater (or equal) than two has a subrepresentation of dimension two. The irreducibility assumption allows us to conclude our claim.

From Lemma 2.10 it is now clear that

$$
\mathcal{L}(\epsilon)=\mathcal{L}_{\bar{\psi}}\left(\operatorname{Res}_{\mathcal{G}}^{G} \epsilon\right)=\mathcal{L}(\mathbf{1})
$$

where $\mathbf{1}$ is the trivial representation. But this is not compatible with the interpolation property in Conjecture 1.3 because the periods $\Omega_{+}$and $\Omega_{-}$are interchanged. This is clear from an philosophical point of view as the periods $\Omega_{ \pm}$arise from paths $\gamma_{ \pm} \in$ $\mathrm{H}_{1}(E(\mathbb{C}), \mathbb{Z})^{ \pm} \subseteq \mathrm{H}_{1}\left(E(\mathbb{C}), \mathbb{Z}_{p}\right)^{ \pm} \cong T_{p} E$, while the period $\Omega_{\infty}$ corresponds to a choice of $\gamma \in T_{\pi} E$ (via the same identification). Thus we define the following correction term which describes the change of complex periods

$$
\mathcal{L}_{\Omega}:=\frac{\Omega_{+}}{\Omega_{\infty}} \frac{1+c}{2}+\frac{\Omega_{-}}{\Omega_{\infty}} \frac{1-c}{2}
$$

Lemma 2.11. $\mathcal{L}_{\Omega} \in \Lambda_{\mathbb{Z}_{p}}(\mathcal{G})^{\times}$.

Proof. In order to prove this lemma we need to understand the relation between the Neron periods $\Omega_{+}, \Omega_{-}$and the period $\Omega_{\infty}$, and in particular show that the ratios $\frac{\Omega_{+}}{\Omega_{\infty}}$ and $\frac{\Omega_{-}}{\Omega_{\infty}}$ belong to $\mathbb{Z}_{p}{ }^{\times}$. Then we have that $\mathcal{L}_{\Omega} \in \Lambda_{\mathbb{Z}_{p}}(\mathcal{G})$ and it is easily seen then that the element $\frac{\Omega_{\infty}}{\Omega_{+}} \frac{1+c}{2}+\frac{\Omega_{\infty}}{\Omega_{-}} \frac{1-c}{2} \in \Lambda_{\mathbb{Z}_{p}}(\mathcal{G})$ is its inverse. Recall that we write $\Lambda$ for the lattice associated to $E$ and we have defined $\Omega_{\infty}$ by $\Lambda=\Omega_{\infty} \mathfrak{f}: \psi$. For our aims we may actually assume that $\Lambda=\Omega_{\infty} O_{K}$ as $\mathfrak{f}=(f)$ for some $f \in K \hookrightarrow K_{\mathfrak{p}} \cong \mathbb{Z}_{p}$ which is a $p$-adic unit since $(\mathfrak{f}, \mathfrak{p})=1$. Recall that we view $O_{K}=\mathbb{Z}+\mathbb{Z} \sqrt{-d_{K}}$ as a lattice in $\mathbb{C}$ by our fixed embedding $K \hookrightarrow \mathbb{C}$.

It is a well-known fact that the Neron periods have the property that $\tau:=\frac{\Omega_{-}}{\Omega_{+}}$is a totally imaginary number and moreover the lattice $\Lambda^{\prime}:=\mathbb{Z} \Omega_{+}+\mathbb{Z} \Omega_{-}$is isomorphic to $\Lambda$ as $\mathbb{Z}\left[\frac{1}{2}\right]$-lattices. As $p \neq 2$ we may work for our purposes with the lattice $\Lambda^{\prime}$ which we keep denoting by $\Lambda$. As the lattice $\Lambda$ has $C M$ by $O_{K}$ we have that $\frac{1}{\Omega_{+}} \Lambda \subseteq K$ is a fractional ideal of $K$ [26, page 164]. In particular we have that $\tau \in K$ and that $\mathbb{Z}+\mathbb{Z} \tau=O_{K} \alpha$ for some $\alpha \in K$. We write $\tau=s \sqrt{-d_{K}}$ and $\alpha=\alpha_{1}+\alpha_{2} \sqrt{-d_{K}}$ with $s, \alpha_{1}, \alpha_{2} \in \mathbb{Q}$. We will show that $s \in \mathbb{Z}_{(p)}^{\times}$and $\alpha \in O_{\mathfrak{p}}^{\times}$. Note that this is enough for our purposes because we have

$$
\mathbb{Z}+\mathbb{Z} \tau=\mathbb{Z}+\mathbb{Z} \frac{\Omega_{-}}{\Omega_{+}}=\frac{\Lambda}{\Omega_{+}}=\frac{\Omega_{\infty}}{\Omega_{+}} O_{K} .
$$

The lattice $\mathbb{Z}+\mathbb{Z} \tau$ has $C M$ by $O_{K}$. That means that we have that

$$
O_{K}=\mathbb{Z}+\mathbb{Z} \sqrt{-d_{K}}=\left\{a+b \tau \mid a, b \in \mathbb{Z}, 2 b r \in \mathbb{Z} \text { and } b\left(r^{2}+d_{K} s^{2}\right) \in \mathbb{Z}\right\} .
$$

As $\sqrt{-d_{K}}$ should belong to the set on the right we have that $b s=1$ and $d_{K} s \in \mathbb{Z}$. That is, $s=\frac{s^{\prime}}{d_{K}}$ for some $s^{\prime} \in \mathbb{Z}$. As $b=\frac{1}{s}=\frac{d_{K}}{s^{\prime}} \in \mathbb{Z}$ we conclude that $s^{\prime} \mid d_{K}$. Hence $s=\left(\frac{d_{K}}{s^{\prime}}\right)^{-1} \in \mathbb{Z}_{p}^{\times}$as $D \in \mathbb{Z}_{p}^{\times}$. That is, $\tau \in O_{\mathfrak{p}}^{\times}$.

Now we show that $\alpha \in O_{\mathfrak{p}}^{\times}$. We have $\mathbb{Z}+\mathbb{Z} \tau=O_{K} \alpha$. We take the completion at $\mathfrak{p}$. It is enough to show that $(\mathbb{Z}+\mathbb{Z} \tau) \otimes_{O_{K}} O_{\mathfrak{p}}=O_{\mathfrak{p}}$. This follows trivially from the fact that $\tau \in O_{\mathfrak{p}}^{\times}$. Hence we obtain that also $\alpha$ is a unit. 
Finally we set

$$
\mathcal{L}_{E}^{\prime}:=\mathcal{L L}_{\Omega}^{-1} \in K_{1}\left(\Lambda_{D}(\mathcal{G})_{S^{*}}\right) .
$$

Proposition 2.12. $\mathcal{L}_{E}^{\prime}$ satisfies the interpolation property in Conjecture 1.3.

Proof. We calculate the fraction $\frac{\mathcal{L}(\breve{\rho})}{\mathcal{L}_{E}^{\prime}(\breve{\rho})}$, where the denominator here is the right hand side of the desired interpolation formula in Conjecture 1.3, in a purely formal way in order to compare all terms showing up, in particular the possible presence of zeroes does not matter at all.

We start with representations $\rho=\operatorname{Ind}_{\mathcal{G}}^{G} \chi$ of type B. Then $\check{\rho}_{\left.\right|_{G}}=\bar{\chi} \oplus \bar{\chi}^{c}$ where $\chi^{c}(g):=\chi\left(c g c^{-1}\right)$ with complex conjugation $c \in \mathcal{G}$.

$$
\begin{gathered}
\frac{\mathcal{L}(\check{\rho})}{\mathcal{L}_{E}^{\prime}(\check{\rho})}=\frac{\mathcal{L}_{\bar{\psi}}(\bar{\chi}) \cdot \mathcal{L}_{\bar{\psi}}\left(\bar{\chi}^{c}\right)}{\mathcal{L}_{E}^{\prime}(\check{\rho})}=\frac{\frac{1}{\Omega_{\infty}^{2}}}{\Omega_{+}^{-1} \Omega_{-}^{-1}} \frac{u^{-\left(\mathfrak{f}_{\bar{p}}(\bar{\chi})+\mathfrak{f}_{\bar{p}}\left(\bar{\chi}^{c}\right)\right)} e_{\overline{\mathfrak{p}}}(\bar{\chi}) e_{\overline{\mathfrak{p}}}\left(\bar{\chi}^{c}\right)}{u^{-\mathfrak{f}_{p}(\check{\rho})} e_{p}(\check{\rho})} \\
\frac{P_{\mathfrak{p}}\left(\bar{\chi}, u^{-1}\right) P_{\overline{\mathfrak{p}}}\left(\chi, u^{-1}\right) P_{\mathfrak{p}}\left(\bar{\chi}^{c}, u^{-1}\right) P_{\overline{\mathfrak{p}}}\left(\chi^{c}, u^{-1}\right)}{P_{p}\left(\check{\rho}, u^{-1}\right) P_{p}\left(\rho, w^{-1}\right)^{-1} P_{p}\left(E, \rho, \frac{1}{p}\right)} \frac{L(\bar{\psi} \chi, 1) L\left(\bar{\psi} \chi^{c}, 1\right)}{L(E, \rho, 1)} \\
=\frac{\Omega_{+} \Omega_{-}}{\Omega_{\infty}} \frac{u^{-\left(\mathfrak{f}_{\mathfrak{p}}(\bar{\chi})+\mathfrak{f}_{\bar{p}}(\bar{\chi})\right)} e_{\mathfrak{p}}(\bar{\chi}) e_{\overline{\mathfrak{p}}}(\bar{\chi})}{u^{-\mathfrak{f}_{p}(\check{\rho})} e_{p}(\check{\rho})} \\
\frac{P_{\mathfrak{p}}\left(\bar{\chi}, u^{-1}\right) P_{\overline{\mathfrak{p}}}\left(\bar{\chi}, u^{-1}\right) P_{\mathfrak{p}}\left(\chi^{c}, u^{-1}\right) P_{\overline{\mathfrak{p}}}\left(\chi^{c}, u^{-1}\right)}{P_{\bar{p}}\left(\bar{\psi} \chi, \frac{1}{p}\right) \quad P_{\overline{\mathfrak{p}}}\left(\bar{\psi} \chi^{c}, \frac{1}{p}\right) P_{\mathfrak{p}}\left(\bar{\psi} \chi^{c}, \frac{1}{p}\right) P_{\mathfrak{p}}\left(\bar{\psi} \chi, \frac{1}{p}\right)} \\
P_{p}\left(\check{\rho}, u^{-1}\right) \\
\text { with } \quad \frac{L(\bar{\psi} \chi, 1) \cdot L\left(\bar{\psi} \chi^{c}, 1\right)}{L((\operatorname{Ind} \bar{\psi}) \otimes \operatorname{Ind} \chi, 1)}=\alpha^{2} \tau \\
\tau:=\frac{\Omega_{-}}{\Omega_{+}}, \quad \alpha:=\frac{\Omega_{+}}{\Omega_{\infty}} \in \mathcal{O}_{\mathfrak{p}}^{\times} \cap K .
\end{gathered}
$$

Let us comment on the calculations above. We start with our considerations on the $\epsilon$-factors. Note that as the representation $\check{\rho}$ is induced from $\bar{\chi}$ we have for the conductor of $\check{\rho}, N_{\check{\rho}}=D N_{K / \mathbb{Q}}\left(N_{\bar{\chi}}\right)$ where $N_{\bar{\chi}}$ the conductor of $\bar{\chi}$. Locally at $p$ that means $\mathfrak{f}_{p}(\check{\rho})=\mathfrak{f}_{\mathfrak{p}}(\bar{\chi})+\mathfrak{f}_{\overline{\mathfrak{p}}}(\bar{\chi})$ as $p$ splits in $K$. Concerning the $\epsilon$-factors note that we know that they are inductive in degree zero. In particular we have

$$
e_{p}\left(\operatorname{Ind}_{G}^{\mathcal{G}}(\bar{\chi} \ominus 1)\right)=e_{\mathfrak{p}}(\bar{\chi} \ominus 1) e_{\overline{\mathfrak{p}}}(\bar{\chi} \ominus 1)=\frac{e_{\mathfrak{p}}(\bar{\chi}) e_{\overline{\mathfrak{p}}}(\bar{\chi})}{e_{\mathfrak{p}}(1) e_{\overline{\mathfrak{p}}}(1)} .
$$

But also

$$
e_{p}\left(\operatorname{Ind}_{G}^{\mathcal{G}}(\bar{\chi} \ominus 1)=\frac{e_{p}\left(\operatorname{Ind} d_{G}^{\mathcal{G}}(\bar{\chi})\right)}{e_{p}(1 \oplus \epsilon)}=\frac{e_{p}(\check{\rho})}{e_{p}(1) e_{p}(\epsilon)}\right.
$$

where as always $\epsilon$ is the non-trivial character of $\operatorname{Gal}(K / \mathbb{Q})$. Hence we obtain

$$
e_{p}(\check{\rho})=\frac{e_{\mathfrak{p}}(\bar{\chi}) e_{\overline{\mathfrak{p}}}(\bar{\chi})}{e_{\mathfrak{p}}(1) e_{\overline{\mathfrak{p}}}(1)} e_{p}(1) e_{p}(\epsilon) .
$$


But note that $p$ split in $K$ we have $e_{p}(1)=e_{p}(\epsilon)=e_{\mathfrak{p}}(1)=e_{\bar{p}}(1)=1$. Hence $e_{p}(\check{\rho})=e_{\mathfrak{p}}(\bar{\chi}) e_{\overline{\mathfrak{p}}}(\bar{\chi})$. Now we explain the ratio of the Euler factors. The starting point is the inductive properties of the Euler factors. In particular as $p$ splits in $K$ we have that $P_{p}(\rho, X)=P_{\mathfrak{p}}(\chi, X) P_{\overline{\mathfrak{p}}}(\chi, X)$. Using this and the relations

$$
u^{-1}=\frac{\psi(\mathfrak{p})}{p}=\frac{\bar{\psi}(\overline{\mathfrak{p}})}{p}, \quad w^{-1}=\frac{\psi(\overline{\mathfrak{p}})}{p}=\frac{\bar{\psi}(\mathfrak{p})}{p},
$$

we see that the Euler factors cancel (the Euler factors which are opposite to each other with respect to the fraction line cancel each other). Finally for the last equation we use the inductiveness of $L$-functions and the following well-known fact from representation theory implied by Frobenius reciprocity

$$
(\operatorname{Ind} \bar{\psi}) \otimes \operatorname{Ind} \chi=\operatorname{Ind}(\bar{\psi} \otimes \operatorname{Res} \operatorname{Ind} \chi)=\operatorname{Ind}\left(\bar{\psi} \otimes\left(\chi \oplus \chi^{c}\right)\right) .
$$

Note that, since $\bar{\psi}=\psi^{c}$ by [17, page 559$]$ also $E=\operatorname{Ind} \bar{\psi}$ holds.

Now we turn to those $\rho$ of type A, i.e $\rho$ is now one dimensional. We have

$$
\begin{aligned}
& \frac{\mathcal{L}(\check{\rho})}{\mathcal{L}_{E}^{\prime}(\check{\rho})}=\frac{\mathcal{L}_{\bar{\psi}}\left(\bar{\rho}_{\mid G}\right)}{\mathcal{L}_{E}^{\prime}(\check{\rho})}= \\
& \frac{\frac{1}{\Omega_{\infty}}}{\Omega_{\rho(c)}^{-1}} \frac{u^{-\mathfrak{f}_{\bar{p}}\left(\bar{\rho}_{\mid G}\right)} e_{\overline{\mathfrak{p}}}\left(\bar{\rho}_{\mid G}\right)}{u^{-\mathfrak{f}_{p}(\bar{\rho})} e_{p}(\bar{\rho})} \frac{P_{\mathfrak{p}}\left(\bar{\rho}_{\mid G}, u^{-1}\right) P_{\overline{\mathfrak{p}}}\left(\bar{\rho}_{\mid G}, u^{-1}\right) P_{p}\left(\rho, w^{-1}\right)}{P_{p}\left(\bar{\rho}, u^{-1}\right) P_{p}\left(E, \rho, p^{-1}\right)} \frac{L\left(\bar{\psi} \rho_{\mid G}, 1\right)}{L(E, \rho, 1)}= \\
& \frac{\Omega_{\rho(c)}}{\Omega_{\infty}} \frac{u^{-\mathfrak{f}_{\mathfrak{p}}\left(\bar{\rho}_{\mid G}\right)} e_{\overline{\mathfrak{p}}}\left(\bar{\rho}_{\mid G}\right)}{u^{-\mathfrak{f}_{p}(\bar{\rho})} e_{p}(\bar{\rho})} \frac{P_{\mathfrak{p}}\left(\bar{\rho}_{\mid G}, u^{-1}\right) P_{\overline{\mathfrak{p}}}\left(\bar{\rho}_{\mid G}, u^{-1}\right) P_{\mathfrak{p}}\left(\rho_{\mid G}, w^{-1}\right)}{P_{\mathfrak{p}}\left(\bar{\rho}_{\mid G}, u^{-1}\right) P_{\mathfrak{p}}\left(\bar{\psi} \rho_{\mid G}, p^{-1}\right) P_{\overline{\mathfrak{p}}}\left(\bar{\psi} \rho_{\mid G}, p^{-1}\right)} \frac{L\left(\bar{\psi} \rho_{\mid G}, 1\right)}{L(E, \rho, 1)}= \\
& \frac{\Omega_{\rho(c)}}{\Omega_{\infty}} \frac{L\left(\bar{\psi} \rho_{\mid G}, 1\right)}{L\left(\operatorname{Ind}\left(\bar{\psi} \otimes \rho_{\mid G}\right), 1\right)}=\left\{\begin{array}{lll}
\alpha & & \rho(c)=+1 \\
& \text { if } & \\
\alpha \tau & & \rho(c)=-1
\end{array} .\right.
\end{aligned}
$$

Recall that $\tau=\frac{\Omega_{-}}{\Omega_{+}}, \alpha=\frac{\Omega_{+}}{\Omega_{\infty}}, \alpha \tau=\frac{\Omega_{-}}{\Omega_{\infty}}$ all belong to $\mathcal{O}_{\mathfrak{p}}^{\times} \cap K$. Let us comment on the above computations. We start with the $\epsilon$-factors. First note that as the extension $K$ is unramified at $p$ we have that $\mathfrak{f}_{p}(\bar{\rho})=\mathfrak{f}_{\mathfrak{p}}\left(\bar{\rho}_{\mid G}\right)$ since as adelic characters $\bar{\rho}_{\mid G}=\bar{\rho} \circ N_{K / \mathbb{Q}}$ and $p$ splits in $K$, that is locally when we identify $K_{\overline{\mathfrak{p}}}$ with $\mathbb{Q}_{p}$ the two characters are equal. This explains also the equality $e_{p}(\bar{\rho})=e_{\overline{\mathfrak{p}}}\left(\bar{\rho}_{\mid G}\right)$. Note that also this remark explains the equality of Euler factors $P_{p}(\rho, X)=P_{\mathfrak{p}}\left(\rho_{\mid G}, X\right)$ and similarly for $\bar{\rho}$. The rest is just trivial inspection of the formula. Finally for the $L$-functions we use as before Frobenius reciprocity and inductive properties of $L$-functions.

Since it is easily checked that

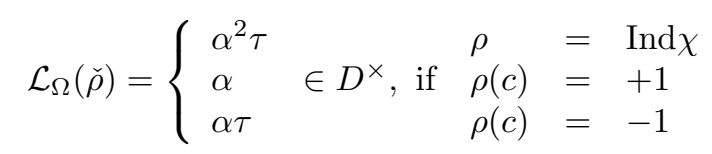

for irreducible $\rho$, we obtain that

$$
\mathcal{L}_{E}^{\prime}(\check{\rho}) \mathcal{L}_{\Omega}(\check{\rho})=\mathcal{L}(\check{\rho})
$$


for all Artin representations $\rho$ as desired. $\square$

Now let $K\left(F_{\infty}\right)$ be the maximal abelian extension of $\mathbb{Q}$ inside $F_{\infty}=K_{\infty}$ in which $p$ does not ramify. We are grateful to John Coates for providing us the proof of the following lemma.

LEMMA 2.13. In our setting we have $K\left(F_{\infty}\right)=K$.

Proof. Since $K\left(F_{\infty}\right)$ is unramified at $p$ we have that it must intersect the composite of all $\mathbb{Z}_{p}$-extensions of $K$ in $K$, and hence we must have that $K\left(F_{\infty}\right)$ is an extension of $K$ contained in $K(E[p])$. If we write $p=\mathfrak{p} \overline{\mathfrak{p}}$ in $K$, the formal group of $E$ at $\mathfrak{p}$ is a Lubin-Tate group, and similarly for the formal group of $E$ at $\overline{\mathfrak{p}}$. Thus $\mathfrak{p}$ is totally ramified in the extension $K(E[\mathfrak{p}]) / K$ which has degree $p-1$, and $\overline{\mathfrak{p}}$ is unramified in this extension. The analogous statement holds for $K(E[\bar{p}]) / K$ but with $\mathfrak{p}$ and $\overline{\mathfrak{p}}$ interchanged. Let $d:=\left[K\left(F_{\infty}\right): K\right]$. Thus we must show that $d=1$.

Now by the definition of $K\left(F_{\infty}\right), \mathfrak{p}$ is unramified in it, and so $K\left(F_{\infty}\right)(E[\mathfrak{p}])$ is an extension of $K$ of degree $d(p-1)$, in which every prime of $K\left(F_{\infty}\right)$ above $\mathfrak{p}$ is totally ramified of ramification index equal to $p-1$ and every prime above $\overline{\mathfrak{p}}$ is unramified. Hence $K\left(F_{\infty}\right)(E[\mathfrak{p}], E[\overline{\mathfrak{p}}])$ must have degree $p-1$ over $K\left(F_{\infty}\right)(E[\mathfrak{p}])$ because every prime of this latter field above $\overline{\mathfrak{p}}$ must be totally ramified of ramification index equal to $p-1$. But $K\left(F_{\infty}\right)(E[\mathfrak{p}], E[\overline{\mathfrak{p}}])=K(E[p])$ and $K(E[p])$ has degree $(p-1)^{2}$ over $K$. Hence $d=1$, and the proof is complete.

So in our setting we have that $K\left(F_{\infty}\right)=K$. Since $p$ splits in $K$ we can identify $K_{\mathfrak{p}}$ with $\mathbb{Q}_{p}$. Then we have,

Theorem 2.14. Assuming Conjecture 1.2 there exists $\mathcal{L}_{E} \in K_{1}\left(\Lambda_{\mathbb{Z}_{p}}(\mathcal{G})_{S}\right)$ satisfying Conjecture 1.3 and 1.4.

Proof. This follows from the explanation before Lemma 2.10 and Theorem 4.3 in the appendix. Indeed assumption (ii) holds because $\mathbb{Z}_{p}(\rho)$ is contained in $\mathbb{Z}_{p}[\mu(p)]$ as the values of $\chi$ (and thus $\rho=\operatorname{Ind} \chi$ or $\chi$ ) are in $\mu_{(p-1) p^{\infty}}$; (iii) is clear from the construction, (iv) follows from the Lemma below while (v) again follows from the explanation before Lemma 2.10.

Lemma 2.15 (Deligne-Conjecture, Blasius). In the CM setting the following algebraicity result holds:

$$
\frac{L_{\{p\}}(E, \rho, 1)}{\Omega_{+}^{d^{+}(\rho)} \Omega_{-}^{d^{-}(\rho)}} e_{p}(\check{\rho}) \frac{P_{p}\left(\stackrel{\vee}{\rho}, u^{-1}\right)}{P_{p}\left(\rho, w^{-1}\right)} u^{-\mathfrak{f}_{p}(\check{\rho})} \in K_{\mathfrak{p}}(\rho) .
$$

Moreover these values are -adically integral.

Proof. That the values are $p$-integral follows from the fact that are obtained as values of a $p$-integral valued measure. So we need to prove that actually the values are in $K_{\mathfrak{p}}(\rho)$ and of course it is enough to prove it for $\rho$ an irreducible Artin representation. Note that as $p$ splits we can identify $\mathbb{Q}_{p}=K_{\mathfrak{p}}$. Moreover we have that $u, w \in \mathbb{Q}_{p}$ hence we need simply to prove that

$$
\frac{L(E, \rho, 1)}{\Omega_{+}^{d^{+}(\rho)} \Omega_{-}^{d^{-}(\rho)}} e_{p}(\check{\rho}) \in K(\rho) .
$$

If $\rho$ is one dimensional then this is well known. Hence we consider the case where $\rho=\operatorname{Ind} d_{\mathcal{G}}^{G} \chi$. Then the above statement is equivalent to

$$
\frac{L(E / K, \chi, 1)}{\Omega_{\infty}^{2}}\left(e_{\mathfrak{p}}(\chi) e_{\overline{\mathfrak{p}}}(\chi)\right)^{-1} \in K(\chi)
$$


using the fact the $\Omega_{+}=\Omega_{-}=\Omega_{\infty}$ up to elements in $K^{\times}, e_{\mathfrak{p}}(\bar{\chi}) e_{\bar{p}}(\bar{\chi})=e_{p}(\check{\rho})$ and the duality of the $\epsilon$-factors. Moreover we have $L(E / K, \chi, 1)=L(\psi, \chi, 1) L(\bar{\psi}, \chi, 1)$. From Blasius proof of Deligne's conjecture for Hecke characters (of CM fields) [3] we know that

$$
\frac{L(\psi, \chi, 1)}{\Omega_{\infty}} c(\psi, \chi)^{-1} \in K(\chi), \frac{L(\bar{\psi}, \chi, 1)}{\Omega_{\infty}} c(\bar{\psi}, \chi)^{-1} \in K(\chi)
$$

where $c(\psi, \chi)$ and $c(\bar{\psi}, \chi)$ are defined as in [4] (see page 65 for the definition and page 67 for the factorization of Deligne's periods for the Hecke characters $\psi \chi$ and $\bar{\psi} \chi)$. Moreover we have that $c(\psi, \chi) c(\bar{\psi}, \chi)=e_{\mathfrak{p}}(\chi) e_{\overline{\mathfrak{p}}}(\chi)$ up to elements in $K(\chi)^{\times}$. This can be seen from the definition of the periods $c(\psi, \chi)$ and $c(\bar{\psi}, \chi)$. From [4, page 65] we have that the element $c(\psi, \chi)$ is an element in $K(\chi) \otimes_{\mathbb{Q}} \overline{\mathbb{Q}}$ characterized from the reciprocity law

$$
(1 \otimes \tau) c(\psi, \chi)=\left(\chi \circ \operatorname{Ver}_{\psi}\right)(\tau) c(\psi, \chi)
$$

where $V r_{\psi}: G_{\mathbb{Q}} \rightarrow G_{K}^{a b}$ is the half-transfer map of Tate associated to the CM-type of the character $\psi$. As it is explained by Blasius this reciprocity law characterizes the element $c(\psi, \chi)$ up to elements in $K(\chi)^{\times}$. From the properties of the half-transfer map of Tate one has that

$$
\operatorname{Ver}_{\psi}(\tau) \cdot \operatorname{Ver}_{\bar{\psi}}(\tau)=\operatorname{Ver}(\tau), \tau \in G_{\mathbb{Q}}
$$

where $\operatorname{Ver}: G_{\mathbb{Q}} \rightarrow G_{K}^{a b}$ is the classical transfer map. But then as is shown in [4, page $70]$, the product of the all epsilon factors $\prod_{\mathfrak{q}} e_{\mathfrak{q}}(\chi)$ has exactly the same reciprocity law as the product $c(\psi, \chi) c(\bar{\psi}, \chi)$ with respect the operation of $G_{\mathbb{Q}}$ and hence they are equal up to elements in $K(\chi)^{\times}$. The characters $\chi$ that we consider are only ramified at $p$ or at the primes $\mathfrak{q}$ that divide $\mathfrak{f}_{\psi}$. But for the epsilon factors $e_{\mathfrak{q}}(\chi)$ for $\mathfrak{q} \mid \mathfrak{f}_{\psi}$ we have that $e_{\mathfrak{q}}(\chi)=e_{q}(\rho)$ up to elements in $K(\chi)^{\times}$where $q:=\mathfrak{q} \cap \mathbb{Q}$. But moreover it is well known $\left[8\right.$, page 330] that $e_{q}(\rho)=e_{q}(\operatorname{det}(\rho))$ up to element in $\mathbb{Q}(\rho)^{\times}$. But $e_{q}(\operatorname{det}(\rho))$ is a Gauss sum and hence an element in $K(\rho)^{\times}$(see [22, pages 103 and 104]) since $q \neq p$ and we know from the lemma above that the maximal abelian extension of $\mathbb{Q}$ inside $K_{\infty}$ unramified at $p$ is $K$. Hence we conclude that also $e_{\mathfrak{q}}(\chi) \in K(\chi)^{\times}$. Hence putting all the above considerations together we conclude that $e_{\mathfrak{p}}(\chi) e_{\overline{\mathfrak{p}}}(\chi)$ is equal up to elements in $K(\chi)^{\times}$to $c(\psi, \chi) c(\bar{\psi}, \chi)$. $\mathbf{\square}$

We finish this section with a last comment. It seems natural to ask if the measure $\mathcal{L}_{\bar{\psi}}$ has already values in $\mathbb{Z}_{p}$ or could be suitably modified by a unit in the Iwasawa algebra $\Lambda_{D}(G)$ to still interpolate the critical values and take values in $\mathbb{Z}_{p}$. Let us first try to explain why we believe that this measure does not take values in $\mathbb{Z}_{p}$. We recall its interpolation property:

$$
\mathcal{L}_{\bar{\psi}}(\bar{\chi})=\int_{G} \bar{\chi} d \mu=\frac{L(\bar{\psi} \chi, 1)}{\Omega_{\infty}} e_{\mathfrak{p}}(\bar{\chi}) P_{\mathfrak{p}}\left(\bar{\chi}, u^{-1}\right) P_{\bar{p}}\left(\chi, u^{-1}\right) u^{-\mathfrak{f}_{\mathfrak{p}}(\bar{\chi})}
$$

for all Artin characters $\chi$ of $G$. Now we note that in order to prove that this measure lies in $\Lambda_{\mathbb{Z}_{p}}(G)$ is equivalent to show the following rationality result

$$
\frac{L(\bar{\psi} \chi, 1)}{\Omega_{\infty}} e_{\mathfrak{p}}(\chi)^{-1} \in K(\chi)
$$


since we are in the ordinary case i.e. $K_{\mathfrak{p}}=\mathbb{Q}_{p}$. As we explained in the proof above we know from Blasius work that

$$
\frac{L(\bar{\psi} \chi, 1)}{\Omega_{\infty}} c(\bar{\psi}, \chi)^{-1} \in K(\chi)
$$

hence one needs to understand if $e_{\mathfrak{p}}(\chi)=c(\bar{\psi}, \chi)$ up to elements in $K(\chi)^{\times}$for finite characters $\chi$ of $G$. However this cannot be the case for all finite characters of $\operatorname{Gal}\left(K^{a b} / K\right)$. (However we remark that it is the case when $\chi$ is cyclotomic i.e. $\chi^{c}=\chi$.) Indeed from Blasius [4] (page 66) we have that the extension of $K$ defined by adjoining to $K$ the values $c(\bar{\psi}, \chi))$ for all finite order characters of $\operatorname{Gal}\left(K^{a b} / K\right)$ is an abelian extension of $K$ not included in $\mathbb{Q}^{a b}$. However the $\epsilon$-factors are just Gauss sums hence they can generate only extensions in $\mathbb{Q}^{a b}$. In particular we have that the two "periods" cannot be equal up to elements in $K(\chi)^{\times} \subseteq \mathbb{Q}^{a b}$ (see also the comment in [22] page 109). Concerning the other question one may speculate that there is a measure $\mathcal{L}_{\bar{\psi}}^{?}$ of $G$ such that

$$
\mathcal{L}_{\bar{\psi}}^{?}(\bar{\chi})=\int_{G} \bar{\chi} d \mu=\frac{L(\bar{\psi} \chi, 1)}{c_{p}(\bar{\psi}, \chi) \Omega_{\infty}} P_{\mathfrak{p}}\left(\bar{\chi}, u^{-1}\right) P_{\overline{\mathfrak{p}}}\left(\chi, u^{-1}\right) u^{-\mathfrak{f}_{\mathfrak{p}}(\bar{\chi})}
$$

for some canonically normalized element $c_{p}(\bar{\psi}, \chi)$ equal to $c(\bar{\psi}, \chi)$ up to elements in $K(\chi)^{\times}$. This measure would have values in $\mathbb{Z}_{p}$.

3. CM-modular forms. In this section we would like to indicate how most of our results can be extended to the case of CM-modular forms. Our reference for the theory of CM-modular forms is Ribet's aricle [19] as well as the last section of Schappacher's book [22].

We start by fixing our setting. Let $f$ be cuspidal newform of weight $k \geq 2$, Nebentypus $\epsilon_{f}$ and level $N$. We pick a number field $F$ that contains all $a_{n}$ of $f=$ $\sum_{n \geq 1} a_{n} q^{n}$. We want now to formulate a $G L_{2}$-Main Conjecture for $f$. Our starting point is the following theorem.

Theorem 3.1 (Eichler-Shimura-Deligne-Scholl-Jannsen). There exists a motive $M(f)$ defined over $\mathbb{Q}$ with coefficients in $F$ of rank two over $F$ such that

$$
L_{N}^{*}(M(f), s):=\left(\prod_{(p, N)=1} \operatorname{det}_{F}\left(1-F r_{p} p^{-s} \mid H_{\ell}(M(f))^{-1}\right)_{\tau}=\left(\sum_{(n, N)=1} a_{n}^{\tau} n^{-s}\right)_{\tau}\right.
$$

where $\tau \in \operatorname{Hom}(F, \mathbb{C}), \ell \neq p$ prime, $F r_{p}$ is a geometric Frobenius element at $p$ and $\operatorname{Re}(s) \gg 0$

Proof. We refer to the book of Schappacher [22] in page 139 and the references there. $\square$

It is known [16, page 240,14.10] that the Kummer dual $M(f)^{*}(1)$ of $M(f)$ is isomorphic to $M\left(f^{*}\right)(k)$, where $f^{*}=\sum_{n \geq 1} \overline{a_{n}} q^{n}$ is the dual cusp form.

We fix an embedding $\tau: F \hookrightarrow \overline{\mathbb{Q}}$, we let $p \geq 5$ be a rational prime and let $\lambda$ be the prime of $F$ above $p$ corresponding to our fixed embedding $F \hookrightarrow \overline{\mathbb{Q}} \hookrightarrow \overline{\mathbb{Q}}_{p}$. We write $\rho_{\lambda}:=\rho_{f, \lambda}: G_{\mathbb{Q}} \rightarrow G L_{2}\left(F_{\lambda}\right)$ for the associated $G_{\mathbb{Q}^{-}}$representation given by the $\lambda$-adic realisation $V:=V(f):=V_{F_{\lambda}}(f)$ of $M(f)$. Moreover we assume that $p$ is a good ordinary prime for $M(f)$, this is equivalent to $p$ being relative prime to $N$ and $a_{p}$ a $\lambda$-adic unit. Note that with $f$ also $f^{*}$ is good ordinary at $p$, see [16, prop. 17.1]. 
We consider the $p$-adic Lie extension $F_{\infty}$ of $\mathbb{Q}$ determined by the image of $\rho_{\lambda}$, i.e. $\mathcal{G}:=\operatorname{Gal}\left(F_{\infty} / \mathbb{Q}\right) \cong \operatorname{Im}\left(\rho_{\lambda}\right)$. We note that the determinant of $\rho_{\lambda}$ is of the form

$$
\operatorname{det}\left(\rho_{\lambda}\right) \cong \chi_{c y c l}^{1-k} \epsilon_{f}^{-1}
$$

and hence $\mathcal{G}$ contains a closed normal subgroup $\mathcal{H}$ such that $\mathcal{G} / \mathcal{H} \cong \mathbb{Z}_{p}$. In particular the setting of Theorem 1.1 of the introduction apply to the above defined group $\mathcal{G}$.

Next we define the $\lambda$-primary Selmer group attached to $f$ as follows: By $V^{\prime}(f)$ we denote the (unique) unramified one dimensional $G_{\mathbb{Q}_{p}}$-subrepresentation of $V(f)$ (restricted to $G_{\mathbb{Q}_{p}}$ ), which exists due to [16, prop. 17.1] $f$ being good ordinary at $\lambda$. We fix an $\mathcal{O}:=\mathcal{O}_{F_{\lambda}}$-lattice $T(f) \subseteq V(f)$ and set $T^{\prime}(f):=T(f) \cap V^{\prime}(f)$ and $T^{\prime \prime}(f):=T(f) / T^{\prime}(f) \subseteq V^{\prime \prime}(f):=V(f) / V^{\prime}(f)$. Then, for any integer $r$, we define

$$
\begin{aligned}
\operatorname{Sel}^{\text {ord }}\left(T(f)(r) / F_{\infty}\right):= & \operatorname{ker}\left(\mathrm{H}^{1}\left(G_{S_{p}}\left(F_{\infty}\right), T(f)(r) \otimes \mathbb{Q} / \mathbb{Z}\right)\right. \\
& \left.\rightarrow \operatorname{Coind}_{\mathcal{G}}^{\mathcal{G}_{p}} \mathrm{H}^{1}\left(F_{\infty, \nu}, T^{\prime \prime}(f)(r) \otimes \mathbb{Q} / \mathbb{Z}\right)\right)
\end{aligned}
$$

where $G_{S_{p}}\left(F_{\infty}\right)$ denotes the Galois group of the maximal outside $p$ unramified extension of $F_{\infty}$ and $\nu$ is a fixed place of $F_{\infty}$ over $p$. Using the same arguments as in the proof of [16, prop. 17.2] one easily shows that this Selmer group coincides with the Bloch-Kato Selmer group $\operatorname{Sel}_{(1)}\left(T(f)(r) \otimes \mathbb{Q} / \mathbb{Z}, F_{\infty}\right)$ in $[11,4.2 .28]$. Finally, we write

$$
X:=X\left(T(f)(r) / F_{\infty}\right):=\operatorname{Sel}^{\text {ord }}\left(T(f)(r) / F_{\infty}\right)^{\vee}
$$

for its Pontryagin dual. Then the Torsion-Conjecture reads as follows

Conjecture 3.2 (Torsion-Conjecture). For one (and hence any) $r$, the dual of the Selmer group is $S^{*}$-torsion:

$$
X\left(T\left(f^{*}\right)(r) / F_{\infty}\right) \in \mathfrak{M}_{\mathcal{H}}(\mathcal{G}) .
$$

We let $L$ denote the same field as in the introduction and we write $\Lambda(\mathcal{G})$ for the Iwasawa algebra of $\mathcal{G}$ with coefficients in $D:=\mathcal{O}_{L}$. We denote by $\Omega_{ \pm}$the periods of Deligne associated to $M(f)$ with respect to our fixed $\tau \in \operatorname{Hom}(F, \mathbb{C})$, that is determined up to elements in $O_{F}^{\times}$, see below for more details. We set

$$
R=\{p\} \cup\left\{l \neq p \mid \text { the ramification index of } l \text { in } F_{\infty} / \mathbb{Q} \text { is infinite }\right\}
$$

and we define $u \in \mathbb{Z}_{p} \times$ by

$$
1-a_{p} T+p^{k-1} \epsilon_{f}(p) T^{2}=(1-u T)(1-w T) .
$$

Conjecture 3.3 (Existence of $p$-adic $L$-function). There is a $\mathcal{L}_{f} \in K_{1}\left(\Lambda(\mathcal{G})_{S^{*}}\right)$ such that

$$
\mathcal{L}_{f}(\rho)=\frac{L_{R}(M(f) \stackrel{\vee}{\rho}, 1)}{\Omega_{+}^{d^{+}(\rho)} \Omega_{-}^{d^{-}(\rho)}} e_{p}(\rho) \frac{P_{p}\left(\rho, u^{-1}\right)}{P_{p}\left(\stackrel{\vee}{\rho}, w^{-1}\right)} u^{-\mathfrak{f}_{p}(\rho)}
$$

for all Artin representations $\rho$ of $\mathcal{G}$.

And similarly:

Conjecture 3.4 (Main Conjecture). The p-adic L-function $\mathcal{L}_{f}$ is a characteristic element of $X\left(T\left(f^{*}\right)(k-1) / F_{\infty}\right)$ :

$$
\partial \mathcal{L}_{f}=\left[X\left(M(f) / F_{\infty}\right)\right]_{D} .
$$


Actually, the last two conjectures are a consequence of the much more general conjectures in [11]. Indeed, theorem 4.2.22 or 4.2.26 in (loc. cit.) applied to the motive $M=M(f)(1)$ with coefficients in $F$ predicts the existence of $\mathcal{L}_{f}$ such that, for $0 \leq i \leq k-2$, the following more general interpolation property holds (at least for $\left.i+1 \neq \frac{k-1}{2}\right)^{2}$

$$
\mathcal{L}_{f}\left(\rho \kappa^{-i}\right)=\frac{L_{R}(M(f) \stackrel{\vee}{\rho}, i+1)}{\Omega_{+}^{d^{+}(\rho, i)} \Omega_{-}^{d^{-}(\rho, i)}(2 \pi \iota)^{d i}}(i !)^{d} e_{p}(\rho) \frac{P_{p}\left(\rho, u^{-1} p^{i}\right)}{P_{p}\left(\rho, u p^{-i-1}\right)}\left(u^{-1} p^{i}\right)^{\mathfrak{f}_{p}(\rho)}
$$

where $d=\operatorname{dim} \rho$ while $d^{+}(\rho, i)$ and $d^{-}(\rho, i)$ denote the dimension of the part of $\rho$ $\mathrm{n}$ which complex conjugation acts as $(-1)^{i}$ and $(-1)^{i-1}$, respectively. To this end note that the eigenvalue $u$ of the geometric Frobenius automorphism acting on $V^{\prime}(f)$ equals $p \nu$ for the $\nu$ in (loc. cit.) due to the compatibility conjecture $C_{W D}$ in $[10,2.4 .3]$, which is known for modular forms (loc.cit., rem 2.4.6(ii)) and for Artin motives, and that

$$
h(r):=\operatorname{dim} g r^{r}\left((M \otimes \rho)_{d R}\right)= \begin{cases}d, & \text { if } r=-1 \text { or } r=k-2 \\ 0, & \text { otherwise, }\end{cases}
$$

because the de Rham realisation $M(f)(j)_{d R}$ has the following decreasing filtration

$$
M(f)(j)_{d R}^{i}= \begin{cases}M(f)_{d R}, & \text { if } i \leq-j \\ M(f)_{d R}^{1}, & \text { if } 1-j \leq i \leq k-1-j ; \\ 0, & \text { if } k-j \leq i\end{cases}
$$

see $[16, \S 11.3]$. In particular, all the twists $M(f)(j), 1 \leq j \leq k-1$, are critical. Moreover $M(f)$ is pure of weight $k-1$ with Hodge decomposition of type $(k-1,0)+$ $(0, k-1)$. The existence of good basis $\gamma^{+}, \gamma^{-}$and $\delta$ of $M_{B}^{+}, M_{B}^{-}$and the tangent space $t_{M}:=M_{d R} / M_{d R}^{0}$, respectively, in the sense of [11, 4.2.24] follows from [16, 17.5] where the dual situation is discussed, in particular we have chosen $\Omega_{ \pm}=\Omega\left(\gamma^{ \pm}, \delta\right)$ in the notation of [11].

Assuming the conjectures in [11], Conjecture 3.4 is a direct consequence of theorem 4.2.22, proposition 4.3.15/16 in (loc. cit.) (observing that $V^{\prime}(f)$ grants an infinite residue extension of $p$ in $F_{\infty} / \mathbb{Q}$ ) once we have seen that $T$ induces the zero class in $K_{0}\left(\mathfrak{M}_{\mathcal{H}}(\mathcal{G})\right)$. If $f$ is not $\mathrm{CM}$, this follows from proposition 4.3 .17 in (loc. cit.) while in the CM-case we give an argument in the proof of Proposition 3.5.

Now we focus on the case where $f$ is a CM-modular form. We will say that $f$ has CM by a non-trivial (quadratic) Dirichlet character $\epsilon$ if

$$
\epsilon(q) a_{q}=a_{q}
$$

for a set of primes $q$ of density 1 . If we write $K$ for the quadratic extension that corresponds to $\epsilon$ we say that $f$ has CM by $K$. For example in our previous setting if we write $f_{E}$ for the newform that corresponds to $E$ we have that $f_{E}$ has CM by the non-trivial quadratic character $\epsilon$ of $\operatorname{Gal}(K / \mathbb{Q})$ as in that case $a_{q}=0$ for the primes that inert in $K$. From now on our fixed modular form $f$ will have CM by some quadratic field $K$ and we will write $\epsilon$ for the associated character. From [19] (proposition 4.4 and theorem 4.5) we know:

\footnotetext{
${ }^{2}$ Otherwise some Euler factors might be zero and the formula can be rewritten by replacing $R$ by the empty set and replacing $\frac{P_{p}\left(\rho, u^{-1} p^{i}\right)}{P_{p}\left({ }^{v}, u p^{-i-1}\right)}$ by $\left\{P_{L, p}(W, u) P_{L, p}(\hat{W}, u)^{-1}\right\}_{u=1} \cdot P_{L, p}\left(\hat{W}^{*}(1), 1\right)$. $\prod_{l \in B} P_{L, l}(W, 1)$, where $W:=M\left(\rho^{*}\right)_{\lambda}=\left[\rho^{*}\right]_{\lambda} \otimes M_{\lambda}$ and $\hat{W}:=\left[\rho^{*}\right]_{\lambda} \otimes V^{\prime}(\hat{f})(1)$.
} 
(i) The field $K$ is imaginary.

(ii) Let $G:=\operatorname{Gal}\left(F_{\infty} / K\right)$. Then $\rho_{\lambda_{\mid}}$is abelian.

(iii) $f$ is (automorphic)-induced by a Grössencharacter $\psi$ over $K$ of type $(k-1,0)$ and after fixing an embedding of $E$ in $\overline{\mathbb{Q}}$ we have $L(f, s)=L(\psi, s)$. Henceforth we assume that $F$ contains $K\left(\psi\left(\hat{K}^{\times}\right)\right)$, where $\hat{K}$ denotes the finite adeles of $K$, e.g. we can just take $F=K\left(\psi\left(\hat{K}^{\times}\right)\right)$by $[16,15.10]$.

(iv) For the $\lambda$-adic representation attached to $\psi$ we have

$$
\rho_{\left.\lambda\right|_{G}}=\psi_{\lambda} \oplus \psi_{\lambda}^{c}
$$

where $\psi_{\lambda}$ is the $\bar{F}_{\lambda}^{\times}$-valued $\lambda$-adic counterpart of the Grössencharacter $\psi$. In particular $\rho_{\lambda}=\operatorname{In} d_{G}^{\mathcal{G}} \psi_{\lambda}=\operatorname{Ind} d_{G}^{\mathcal{G}} \psi_{\lambda}^{c}$, see also [16, (15.11.2)]. Here our convention is that $\psi_{\lambda}\left(\left(\mathfrak{a}, K_{\infty} / K\right)\right)=\psi(\mathfrak{a})^{-1}$ and we write $V(\psi)=V_{F_{\lambda}}(\psi)$ for the corresponding representation space.

(v) $\psi \psi^{c}=N_{K}^{k-1}\left(\epsilon_{f} \circ N_{K}\right)$.

Assumption. We are going to assume all along that the size of the torsion part of $G$ is relative prime to $p$.

Now we pick a prime $p$ that splits in $K$ and write $p=\mathfrak{p} \overline{\mathfrak{p}}$ for a prime $\mathfrak{p}$ of $K$. We make the standard assumption that $\mathfrak{p}$ is the prime that corresponds to the $p$-adic embedding

$$
K \hookrightarrow \overline{\mathbb{Q}} \hookrightarrow \overline{\mathbb{Q}}_{p}
$$

with respect to our fixed embedding $\overline{\mathbb{Q}} \hookrightarrow \overline{\mathbb{Q}}_{p}$, in particular $\lambda \mid \mathfrak{p}$. We now note that

$$
1-a_{p} T+p^{k-1} \epsilon_{f}(p) T^{2}=(1-\psi(\overline{\mathfrak{p}}) T)(1-\psi(\mathfrak{p}) T)
$$

and claim that $\psi(\overline{\mathfrak{p}})$ is a $\lambda$-adic unit. Indeed, as the character $\psi$ is of type $(k-1,0)$ we have that its $\lambda$-adic counterpart $\psi_{\lambda}$ factors through $\operatorname{Gal}\left(K\left(\mathfrak{f}_{\psi} \mathfrak{p}^{\infty}\right) / K\right)$, i.e. is it is a character of the form

$$
\psi_{\lambda}: \operatorname{Gal}\left(K\left(\mathfrak{f}_{\psi} \mathfrak{p}^{\infty}\right) / K\right) \rightarrow \bar{O}_{\lambda}^{\times}
$$

where $\bar{O}_{\lambda}$ the ring of integers of $\bar{F}_{\lambda}^{\times}$. But then $\psi(\overline{\mathfrak{p}})=\psi_{\lambda}\left(F r o b_{\overline{\mathfrak{p}}}\right) \in \bar{O}_{\lambda}^{\times}$and we have $u=\psi(\overline{\mathfrak{p}})$.

For a $G_{K}$-representation $\rho: G_{K} \rightarrow \operatorname{Aut}(V)$ on a finite dimensional $F_{\lambda}$-vector space $V$ and any Galois stable $\mathcal{O}:=\mathcal{O}_{F_{\lambda}}$-lattice $T \subseteq V$ we define

$$
S\left(T / K_{\infty}\right):=\operatorname{ker}\left(\mathrm{H}^{1}\left(G_{S_{p}}\left(K_{\infty}\right), T \otimes \mathbb{Q} / \mathbb{Z}\right) \rightarrow \operatorname{Coind}_{G}^{G_{\bar{p}}} \mathrm{H}^{1}\left(K_{\infty, \nu}, T \otimes \mathbb{Q} / \mathbb{Z}\right)\right)
$$

where $\nu$ as before denotes any fixed place of $K_{\infty}$ lying over $\overline{\mathfrak{p}}$. Its Pontryagin-dual

$$
\mathcal{X}\left(T / K_{\infty}\right)=S\left(T / K_{\infty}\right)^{\vee} \cong \mathcal{X}\left(K_{\infty}\right) \otimes_{\mathbb{Z}_{p}} T^{*}
$$

is a finitely generated $\Lambda_{\mathcal{O}}(G)$-module. We fix an $\mathcal{O}$-lattice $T(\psi)^{*} \subseteq V(\psi)^{*}$ and assume that

$$
T:=T\left(f^{*}\right)(k-1)
$$

coincides with

$$
\operatorname{Ind}_{\mathbb{Q}}^{K} T(\psi)^{*} \subseteq V(f)^{*}=V\left(f^{*}\right)(k-1) .
$$


As noted above $T(\psi)^{*}$ is unramified at $\overline{\mathfrak{p}}$, whence the free rank one $\mathcal{O}$-module $T\left(\psi^{c}\right)^{*}$ with Galois action given by the complex conjugate character $\left(\psi^{c}\right)^{-1}$ is unramified at $\mathfrak{p}$. It follows immediately that

$$
T\left(\psi^{c}\right)^{*}=T^{\prime}:=T^{\prime}\left(f^{*}\right)(k-1) \text { and } T(\psi)^{*}=T^{\prime \prime}:=T^{\prime \prime}\left(f^{*}\right)(k-1)
$$

as $G_{K}$-modules.

Proposition 3.5. There is a natural isomorphism of $\Lambda(\mathcal{G})$-modules

$$
X\left(T / K_{\infty}\right) \cong \operatorname{Ind}_{\mathcal{G}}^{G}\left(\mathcal{X}\left(K_{\infty}\right) \otimes_{\mathbb{Z}_{p}} T(\psi)\right),
$$

where $G$ acts diagonally on the tensor product.

Proof. Applying the exact functor $\operatorname{Coind}_{\mathcal{G}}^{G}$ to the defining sequence

$0 \rightarrow S\left(T(\psi)^{*} / K_{\infty}\right) \rightarrow \mathrm{H}^{1}\left(G_{S_{p}}\left(K_{\infty}\right), T(\psi)^{*} \otimes \mathbb{Q} / \mathbb{Z}\right) \rightarrow \operatorname{Coind}_{G}^{G_{\bar{p}}} \mathrm{H}^{1}\left(K_{\infty, \nu}, T(\psi)^{*} \otimes \mathbb{Q} / \mathbb{Z}\right)$

using the transitivity of coinduction and the isomorphisms

$$
\begin{aligned}
\operatorname{Coind}_{\mathcal{G}}^{G} \mathrm{H}^{1}\left(G_{S_{p}}\left(K_{\infty}\right), T(\psi)^{*} \otimes \mathbb{Q} / \mathbb{Z}\right) & \cong \operatorname{Hom}\left(G_{S_{p}}\left(K_{\infty}\right), \operatorname{Coind}_{\mathcal{G}}^{G}\left(T(\psi)^{*} \otimes \mathbb{Q} / \mathbb{Z}\right)\right) \\
& \cong H^{1}\left(G_{S_{p}}\left(K_{\infty}\right), T \otimes \mathbb{Q} / \mathbb{Z}\right)
\end{aligned}
$$

as well as

$$
\mathrm{H}^{1}\left(K_{\infty, \nu}, T(\psi)^{*} \otimes \mathbb{Q} / \mathbb{Z}\right) \cong \mathrm{H}^{1}\left(K_{\infty, \nu}, T^{\prime \prime} \otimes \mathbb{Q} / \mathbb{Z}\right),
$$

one just obtains the defining sequence of the full Selmer group

$$
0 \longrightarrow \operatorname{Sel}^{\text {ord }}\left(T / K_{\infty}\right) \rightarrow \mathrm{H}^{1}\left(G_{S_{p}}\left(K_{\infty}\right), T \otimes \mathbb{Q} / \mathbb{Z}\right) \longrightarrow \operatorname{Coind}_{\mathcal{G}}^{\mathcal{G}_{\bar{p}}} \mathrm{H}^{1}\left(K_{\infty, \nu}, T^{\prime \prime} \otimes \mathbb{Q} / \mathbb{Z}\right)
$$

The result now follows by taking duals. Finally, we give the promised proof that the class of $X\left(T / K_{\infty}\right)$ coincides with that of the Selmer complex used in [11]: Let $H^{\prime}$ be the (open) maximal torsionfree pro- $p$ (abelian) subgroup of $H$, i.e. $H=H^{\prime} \times H^{\prime \prime}$ for some finite abelian group $H^{\prime \prime}$. Thus we have a natural functor from the category $\Lambda\left(H^{\prime}\right)$-mod of finitely generated $\Lambda\left(H^{\prime}\right)$-modules to $\mathfrak{M}_{H}(G)$ by extending the $H^{\prime}$ action to a $G$-action letting $G / H^{\prime} \cong H^{\prime \prime} \times \Gamma$ act trivially, which induces the first homomorphism in the following composition

$$
K_{0}\left(\Lambda\left(H^{\prime}\right)-\bmod \right) \rightarrow K_{0}\left(\mathfrak{M}_{H}(G)\right) \rightarrow K_{0}\left(\mathfrak{M}_{H}(G)\right) \rightarrow K_{0}\left(\mathfrak{M}_{\mathcal{H}}(\mathcal{G})\right),
$$

where the second map is induced by twisting with $T(\psi)^{*}$ while the last one is induced by tensoring with $\Lambda(\mathcal{G}) \otimes_{\Lambda(G)}-$. As, $\Lambda\left(H^{\prime}\right)$ being a regular local ring, the $\Lambda\left(H^{\prime}\right)$-rank induces an isomorphism $K_{0}\left(\Lambda\left(H^{\prime}\right)\right.$-mod $) \cong \mathbb{Z}$, one sees that the class of the trivial $H^{\prime}$-module $\mathbb{Z}_{p}$, which is sent to the class of $T=\operatorname{Ind}_{\mathcal{G}}^{G}\left(T(\psi)^{*}\right.$ under the above map, is zero.

Now we explain the construction of the non-abelian $p$-adic $L$-function. We start by fixing archimedean and $p$-adic periods that correspond canonical to the Grössencharacter $\psi$ that we have associated to our CM modular form $f$. We pick a prime ideal $\mathfrak{f}$ of $K$ that is relative prime to $\mathfrak{p}$ and with the property that the integer $w_{\mathfrak{f}}$ defined as the number of roots of unity in $K$ congruent to 1 modulo $f$ is equal to 1. Then from [9] (Lemma in page 41) we know that there is a Grössencharacter $\phi$ 
of $K$ of conductor $\mathfrak{f}$ and type $(1,0)$. Moreover from the same lemma in [9] we know that if $\phi$ is a Grössencharacter of $K$ of type $(1,0)$ then there exists an elliptic curve defined over $K\left(\mathfrak{f}_{\phi}\right)$ where $\mathfrak{f}_{\phi}$ the conductor of $\phi$ such that $E$ has CM by $O_{K}$ and its associated Grössencharacter is given by $\psi_{E}=\phi \circ N_{K\left(\mathfrak{f}_{\phi}\right) / K}$. Now we are ready to define our periods for our Grössencharacter $\psi$. We distinguish two cases

(i) $\psi$ is of type $(1,0)$, i.e. $f$ is of weight 2 . Then we write $E$ for the elliptic curve defined over $K\left(\mathfrak{f}_{\psi}\right)$ and $\Lambda$ for its corresponding lattice in $\mathbb{C}$. Then we define $\Omega_{\infty}$ by

$$
\Omega_{\infty} \Lambda=\mathfrak{f}
$$

where we are implicity assuming that we see $\mathfrak{f}$ as a lattice in $\mathbb{C}$ with respect to the embedding $K \hookrightarrow \mathbb{C}$ imposed by the CM type of the character $\psi$ (see also [9] page 66). The $p$-adic periods $\Omega_{p}$ are defined using the elliptic curve $E$ as is done in [9] page 66)

(ii) If the character $\psi$ is of type $(k-1,0)$ for $k>2$ then we pick some other Grössencharacter $\phi$ of type $(1,0)$ and of conductor $\mathfrak{f}_{\phi}$ prime to $\mathfrak{p}$ and write

$$
\psi=\theta \phi^{k-1}
$$

where $\theta$ is some finite order character of conductor relative prime to $\mathfrak{p}$. We define then the periods $\Omega_{\infty}$ and $\Omega_{p}$ as in (i) using the character $\phi$.

Let $K\left(\mathfrak{p}^{\infty}\right)$ be the maximal $\mathbb{Z}_{p^{-}}$-extension of $K$ inside $K\left(\mathfrak{f}_{\psi} \mathfrak{p}^{\infty}\right)$ and set $G^{\prime}:=$ $\operatorname{Gal}\left(K\left(\mathfrak{f}_{\psi} \mathfrak{p}^{\infty}\right) / K\left(\mathfrak{p}^{\infty}\right)\right)$. Let $m:=\left|G^{\prime}\right|$, then we define $D$ to be the ring generated over $\widehat{\mathbb{Z}_{p}^{n r}}$ by the $m^{\text {th }}$ roots of unity. Using exactly the same construction as in section 2 we conclude the following:

COROLlary 3.6. There exists a unique $\mathcal{L}_{\bar{\psi}}:=\mu \in \Lambda_{D}(G)$ such that for $0 \leq-j$ and $0<k-1+j$

$$
\begin{gathered}
\mathcal{L}_{\bar{\psi}}\left(\chi \kappa^{j}\right)=\int_{G} \chi \kappa^{j} d \mu=\Gamma(k-1+j) i^{k-1+j} \frac{L(\bar{\psi} \chi, k-1+j)}{(2 \pi)^{j} \Omega_{\infty}^{k-1}} \times \\
e_{\mathfrak{p}}(\bar{\chi}) P_{\mathfrak{p}}\left(\bar{\chi}, \frac{w}{p^{-j+1}}\right) P_{\overline{\mathfrak{p}}}\left(\chi, u^{-1} p^{-j}\right)\left(\frac{\psi(\mathfrak{p})}{p}\right)^{\mathfrak{f}_{\mathfrak{p}}(\bar{\chi})} p^{j \mathfrak{f}_{\mathfrak{p}}(\bar{\chi})}
\end{gathered}
$$

for all Artin-character $\chi$ of $G$ and the cyclotomic character $\kappa: F r o b_{\mathfrak{q}}^{-1} \mapsto N_{K}(\mathfrak{q})$.

Proof. We write $\mathfrak{g}$ for the maximal ideal that is contained in $\mathfrak{f}$ and $\overline{\mathfrak{f}}$. From 2.3 we know that there exists a measure $\mu\left(\mathfrak{g} \overline{\mathfrak{p}}^{\infty}\right)$ of $G\left(K\left(\mathfrak{g} p^{\infty}\right) / K\right)$ so that

$$
\Omega_{p}^{j-k} \int_{G\left(K\left(\mathfrak{g} p^{\infty}\right) / K\right)} \epsilon^{-1}(\sigma) \mu\left(\mathfrak{g} \overline{\mathfrak{p}}^{\infty}\right)=\Gamma(k) i^{k} \Omega_{\infty}^{j-k}\left(\frac{\sqrt{d_{K}}}{2 \pi}\right)^{j} G(\epsilon)\left(1-\frac{\epsilon(\mathfrak{p})}{p}\right) L_{\overline{\mathfrak{p}} \mathfrak{g}}\left(\epsilon^{-1}, 0\right)
$$

for $\epsilon$ of type $(k, j)$ with $0 \leq-j$ and $k>0$. As explained above the character $\psi$ is of the form $\phi^{k-1} \theta$ for $\phi$ of type $(1,0)$ and $\theta$ a finite character, both unramified at $p$. Moreover we have the relation $\phi \bar{\phi}=N_{K}$. In particular for a finite order character $\chi$ of $\operatorname{Gal}\left(K\left(p^{\infty}\right) / K\right)$ the character

$$
\epsilon:=\psi N_{K}^{j} \bar{\chi}=\phi^{k-1+j} \bar{\phi}^{j} \theta \bar{\chi}
$$


is a valid choice for $\epsilon$ above provided that $0 \leq-j$ and $0<k-1+j$. But then for the $L$-functions we have the equalities

$$
L\left(\epsilon^{-1}, 0\right)=L\left(\psi^{-1} N_{K}^{-j} \chi, 0\right)=L\left(\bar{\psi} \chi N_{K}^{-(k-1+j)}\right)=L(\bar{\psi} \chi, k-1+j) .
$$

Then the proof is the same as in the case of elliptic curves with CM that we did in corollary 2.6. $\mathrm{Q}$

We note that in the more general case that we consider now we do not have $\bar{\psi}=\psi^{c}$ but only that $\psi^{c}=\bar{\psi}\left(\epsilon_{f} \circ N_{K}\right)$ which means that the motive $M(f)$ is not self-dual. We now use the functional equation in order to get the critical values at the point $s=1$. For the character $\psi \bar{\chi}$ of type $(k-1,0)$ we have that its dual representation is given by the character $(\psi \bar{\chi})^{-1}=\psi^{-1} \chi$ for which we have that $L\left(\psi^{-1} \chi, s\right)=L(\bar{\psi} \chi, s+(k-1))$. Then the functional equation reads (see [29] page 16 or [9] page 37 )

$$
\Gamma_{\psi}(s) L(\psi \bar{\chi}, s)=e\left(\psi \bar{\chi} \omega_{s}\right) \Gamma_{\bar{\psi}}((k-1)+1-s) L(\bar{\psi} \chi,(k-1)+1-s)
$$

where for a character $\phi$ of type $(k, j)$ we write $\Gamma_{\phi}(s):=\frac{\Gamma(s-\min (k, j))}{(2 \pi)^{s-m i n(k, j)}}$ and $e\left(\psi \bar{\chi} \omega_{s}\right)=$ $\prod_{\mathfrak{q}} e_{\mathfrak{q}}\left(\psi \bar{\chi} \omega_{s}, \psi_{a d}, d x_{\psi_{a d}}\right)$ where $\omega_{s}$ as in Tate's [29]. In particular for $s:=1-j$ with $j \leq 0$ we have

$$
L(\bar{\psi} \chi, k-1+j)=\frac{\Gamma_{\psi}(1-j)}{\Gamma_{\bar{\psi}}(k-1+j)} e\left(\psi \bar{\chi} \omega_{1-j}\right)^{-1} L(\psi \bar{\chi}, 1-j) .
$$

Hence

$$
\begin{gathered}
L(\bar{\psi} \chi, k-1+j) e_{\mathfrak{p}}(\bar{\chi})\left(\frac{\psi(\mathfrak{p})}{p}\right)^{\mathfrak{f}_{\mathfrak{p}}(\bar{\chi})} p^{j \mathfrak{f}_{\mathfrak{p}}(\bar{\chi})}= \\
\frac{\Gamma_{\psi}(1-j)}{\Gamma_{\bar{\psi}}(k-1+j)} e\left(\psi \bar{\chi} \omega_{1-j}\right)^{-1} e_{\mathfrak{p}}(\bar{\chi})\left(\frac{\psi(\mathfrak{p})}{p}\right)^{\mathfrak{f}_{\mathfrak{p}}(\bar{\chi})} p^{j \mathfrak{f}_{\mathfrak{p}}(\bar{\chi})} L(\psi \bar{\chi}, 1) .
\end{gathered}
$$

But we have

$$
\begin{gathered}
e\left(\psi \bar{\chi} \omega_{1-j}\right)^{-1} e_{\mathfrak{p}}(\bar{\chi})\left(\frac{\psi(\mathfrak{p})}{p}\right)^{\mathfrak{f}_{\mathfrak{p}}(\bar{\chi})} p^{j \mathfrak{f}_{\mathfrak{p}}(\bar{\chi})}=\prod_{\mathfrak{q}} e_{\mathfrak{q}}\left(\psi \bar{\chi} \omega_{1-j}, \psi_{a d}, d x_{\psi_{a d}}\right)^{-1} e_{\mathfrak{p}}\left(\psi \bar{\chi} \omega_{1-j}\right)= \\
\prod_{\mathfrak{q} \neq \mathfrak{p}} e_{\mathfrak{q}}\left(\psi \bar{\chi} \omega_{1-j}, \psi_{a d}, d x_{\psi_{a d}}\right)^{-1}
\end{gathered}
$$

where we have used the fact that $d x_{\psi_{a d}}=d x_{1}$ at $\mathfrak{p}$ as this prime is unramified in $K$. Now we observe that

$$
\begin{aligned}
e_{\overline{\mathfrak{p}}}\left(\psi \bar{\chi} \omega_{1-j}, \psi\right)=e_{\overline{\mathfrak{p}}}\left(\bar{\chi} \omega_{1-j}, \psi_{a d}, d x_{1}\right) \psi(\overline{\mathfrak{p}})^{\mathfrak{f}_{\overline{\mathfrak{p}}}(\bar{\chi})} & =e_{\overline{\mathfrak{p}}}\left(\chi \omega_{j}, \psi_{a d}^{-1}\right)^{-1} \psi(\overline{\mathfrak{p}})^{\mathfrak{f}_{\overline{\mathfrak{p}}}(\bar{\chi})} \\
& =e_{\overline{\mathfrak{p}}}\left(\chi \omega_{j}, \psi_{a d}^{-1}\right)^{-1} u^{\mathfrak{f}_{\overline{\mathfrak{p}}}(\bar{\chi})}
\end{aligned}
$$

where we have used the duality $e_{\overline{\mathfrak{p}}}\left(\chi \omega_{j}, \psi_{a d}^{-1}, d x_{1}\right) e_{\overline{\mathfrak{p}}}\left(\bar{\chi} \omega_{1-j}, \psi_{a d}, d x_{1}\right)=1$. Hence we obtain

$$
\prod_{\mathfrak{q} \neq \mathfrak{p}} e_{\mathfrak{q}}\left(\psi \bar{\chi} \omega_{1-j}, \psi_{a d}, d x_{\psi_{a d}}\right)^{-1}=\prod_{\mathfrak{q} \neq \mathfrak{p}, \overline{\mathfrak{p}}} e_{\mathfrak{q}}\left(\psi \bar{\chi} \omega_{1-j}, \psi_{a d}, d x_{\psi_{a d}}\right)^{-1} e_{\overline{\mathfrak{p}}}(\chi) u^{-\mathfrak{f}_{\bar{p}}(\bar{\chi})} p^{-j \mathfrak{f}_{\mathfrak{p}}(\bar{\chi})} .
$$


We can now state the following:

Corollary 3.7. There exists a unique $\mathcal{L}_{\bar{\psi}}^{(t w)}:=\mu \in \Lambda_{D}(G)$ such that for $0 \leq-j$ and $0<k-1+j$ we have

$$
\begin{aligned}
& \mathcal{L}_{\bar{\psi}}^{(t w)}\left(\chi \kappa^{j}\right)=\int_{G} \chi \kappa^{j} d \mu=\Gamma(k-1+j) i^{j} \frac{L(\psi \bar{\chi}, 1-j)}{(2 \pi)^{j} \Omega_{\infty}^{k-1}} e_{\overline{\mathfrak{p}}}(\chi) \times \\
& P_{\mathfrak{p}}\left(\bar{\chi}, \frac{w}{p^{-j+1}}\right) P_{\overline{\mathfrak{p}}}\left(\chi, u^{-1} p^{-j}\right) u^{-\mathfrak{f}_{\overline{\mathfrak{p}}}(\bar{\chi})} p^{-j \mathfrak{f}_{\mathfrak{p}}(\bar{\chi})}\left(\frac{\Gamma_{\psi}(1-j)}{\Gamma_{\bar{\psi}}(k-1+j)}\right)
\end{aligned}
$$

for all Artin-character $\chi$ of $G$.

Proof. We have already constructed a measure with the interpolation property

$$
\begin{gathered}
\mathcal{L}_{\bar{\psi}}\left(\chi \kappa^{j}\right)=\int_{G} \chi \kappa^{j} d \mu=\Gamma(k-1+j) \frac{L(\bar{\psi} \chi, k-1+j)}{(2 \pi)^{j} \Omega_{\infty}^{k-1}} e_{\mathfrak{p}}(\bar{\chi}) P_{\mathfrak{p}}\left(\bar{\chi}, \frac{w}{p^{-j+1}}\right) \times \\
P_{\overline{\mathfrak{p}}}\left(\chi, u^{-1} p^{-j}\right)\left(\frac{\psi(\mathfrak{p})}{p}\right)^{\mathfrak{f}_{\mathfrak{p}}(\bar{\chi})} p^{j \mathfrak{f}_{\mathfrak{p}}(\bar{\chi})}
\end{gathered}
$$

and using the above computations we can rewrite it as

$$
\begin{gathered}
\mathcal{L}_{\bar{\psi}}\left(\chi \kappa^{j}\right)=\int_{G} \chi \kappa^{j} d \mu=\left(\frac{\Gamma_{\psi}(1-j)}{\Gamma_{\bar{\psi}}(k-1+j)} \prod_{\mathfrak{q} \neq \mathfrak{p}, \overline{\mathfrak{p}}} e_{\mathfrak{q}}\left(\psi \bar{\chi} \omega_{1-j}, \psi_{a d}, d x_{\psi_{a d}}\right)^{-1}\right) \times \\
\Gamma(k-1+j) i^{j} \frac{L(\psi \bar{\chi}, 1-j)}{(2 \pi)^{j} \Omega_{\infty}^{k-1}} e_{\overline{\mathfrak{p}}}(\chi) P_{\mathfrak{p}}\left(\bar{\chi}, \frac{w}{p^{-j+1}}\right) P_{\overline{\mathfrak{p}}}\left(\chi, u^{-1} p^{-j}\right) u^{-\mathfrak{f}_{\bar{p}}(\bar{\chi})} p^{-j \mathfrak{f}_{\mathfrak{p}}(\bar{\chi})} .
\end{gathered}
$$

Now we claim that the mapping $\chi \kappa^{j} \mapsto \prod_{\mathfrak{q} \neq \mathfrak{p}, \overline{\mathfrak{p}}} e_{\mathfrak{q}}\left(\psi \bar{\chi} \omega_{1-j}, \psi_{a d}, d x_{\psi_{a d}}\right)$ is a unit in the Iwasawa algebra $\Lambda_{D}(G)$ and hence we can twist our measure by this element to conclude the proposition.

We write $K_{\infty} \subseteq K\left(p^{\infty}\right)$ for the $\mathbb{Z}_{p}^{2}$-extension of $K$ and define $\Gamma:=\operatorname{Gal}\left(K_{\infty} / K\right) \cong$ $\mathbb{Z}_{p}^{2}$. We decompose $G=\Delta \times \Gamma$ for $\Delta$ finite of order relative prime to $p$. Then we have that $\Lambda_{D}(G)=D[[G]]=D[\Delta][[\Gamma]]$. We write $\hat{\Delta}$ for the group of characters of $\Delta$. Then by our assumptions on $D$ we have that $D[\Delta] \cong \oplus_{\theta \in \hat{\Delta}} D$ given by $\alpha \mapsto(\ldots, \theta(\alpha), \ldots)$ and hence $\Lambda_{D}(G) \cong \oplus_{\theta} D[[\Gamma]]$. Every Artin character $\chi$ of $G$ can be decomposed as $\chi=\theta \chi_{\Gamma}$ for $\theta$ a character of $\Delta$ and $\chi_{\Gamma}$ a character of $\Gamma$. We write $\mathfrak{d}_{K}$ for the different of $K$ over $\mathbb{Q}$ We note that $\theta$ can be ramified at $p$ or at $\mathfrak{q}$ with $\mathfrak{q} \mid \mathfrak{f}_{\psi}$ for the conductor of $\psi$. We write $\mathfrak{f}_{\psi \theta}$ for the non- $p$ part of the conductor of $\psi \bar{\theta}$. Then we have

$$
\begin{aligned}
\prod_{\mathfrak{q} \neq \mathfrak{p}, \overline{\mathfrak{p}}} e_{\mathfrak{q}}\left(\psi \bar{\chi} \omega_{1-j}, \psi, d x_{\psi}\right) & =i^{k-1} \prod_{\mathfrak{q} \mid \mathfrak{f}_{\psi} \mathfrak{d}_{K}} e_{\mathfrak{q}}\left(\psi \bar{\chi} \omega_{1-j}, \psi_{a d}, d x_{\psi_{a d}}\right) \\
& =\bar{\chi}_{\Gamma}\left(\mathfrak{f}_{\psi \theta} \mathfrak{d}_{K}\right) N_{K}\left(\mathfrak{f}_{\psi \theta} \mathfrak{d}_{K}\right)^{-(1-j)} i^{k-1} \prod_{\mathfrak{q} \mid \mathfrak{f}_{\psi^{\prime}} \mathfrak{d}_{K}} e_{\mathfrak{q}}\left(\psi \bar{\theta}, \psi_{a d}, d x_{\psi_{a d}}\right)
\end{aligned}
$$


since $\chi_{\Gamma}$ is only at $p$ ramified and $\left(p, \mathfrak{f}_{\psi} \mathfrak{d}_{K}\right)=1$ under our assumptions. But $\prod_{\mathfrak{q} \mid \mathfrak{f}_{\psi} \mathfrak{d}_{K}} e_{\mathfrak{q}}\left(\psi \bar{\theta}, \psi_{a d}, d x_{\psi_{a d}}\right)$ is a $\mathfrak{p}$-adic unit (see [9] page 94$)$ and hence we can define the measure

$$
\frac{1}{N_{K}\left(\mathfrak{f}_{\psi \theta} \mathfrak{d}_{K}\right)} \prod_{\mathfrak{q} \mid \mathfrak{f}_{\psi} \mathfrak{d}_{K}} e_{\mathfrak{q}}\left(\psi \bar{\theta}, \psi_{a d}, d x_{\psi_{a d}}\right) \sigma_{\mathfrak{f}_{\psi \theta} \mathfrak{d}_{K}}^{-1} \in \Lambda_{D}[[\Gamma]]^{\times}
$$

where $\sigma_{\mathfrak{f}_{\psi \theta} \mathfrak{d}_{K}} \in \Gamma$ corresponds through the Artin reciprocity to $\mathfrak{f}_{\psi \theta} \mathfrak{d}_{K}$, well defined as $K_{\infty}$ ramifies only at $p$. We then define the element $E \in \Lambda_{D}[[G]]^{\times}$to be the element that corresponds to

$$
\left(\ldots, \frac{1}{N_{K}\left(\mathfrak{f}_{\psi \theta} \mathfrak{d}_{K}\right)} \prod_{\mathfrak{q} \mid \mathfrak{f}_{\psi} \mathfrak{d}_{K}} e_{\mathfrak{q}}\left(\psi \bar{\theta}, \psi_{a d}, d x_{\psi_{a d}}\right) \sigma_{\mathfrak{f}_{\psi \theta} \mathfrak{d}_{K}}^{-1}, \ldots\right) \in \oplus_{\theta} D[[\Gamma]]
$$

under the above mentioned isomorphism $\Lambda_{D}(G) \cong \oplus_{\theta} D[[\Gamma]]$. We then obtain for a character $\chi$ of $G$

$$
E\left(\chi \kappa^{j}\right)=i^{1-k} \prod_{\mathfrak{q} \neq \mathfrak{p}, \overline{\mathfrak{p}}} e_{\mathfrak{q}}\left(\psi \bar{\chi} \omega_{1-j}, \psi, d x_{\psi}\right)
$$

which allows us to conclude the proposition. $\square$

Using now the natural map

$$
\left(\imath_{S}\right)_{*}: K_{1}\left(\Lambda(G)_{S^{*}}\right) \rightarrow K_{1}\left(\Lambda(\mathcal{G})_{S^{*}}\right)
$$

we define

$$
\mathcal{L}:=\left(\imath_{S}\right)_{*}\left(\mathcal{L}_{\bar{\psi}}\right) \text { and } \mathcal{L}^{(t w)}:=\left(\imath_{S}\right)_{*}\left(\mathcal{L}_{\bar{\psi}}^{(t w)}\right)
$$

As in the case of elliptic curves with CM we need to understand the following correction term which describes the change of complex periods

$$
\mathcal{L}_{\Omega}:=\frac{(2 \pi)^{k-2} \Omega_{+}}{\Omega_{\infty}^{k-1}} \frac{1+c}{2}+\frac{(2 \pi)^{k-2} \Omega_{-}}{\Omega_{\infty}^{k-1}} \frac{1-c}{2} .
$$

Conjecture 3.8 (Period Relation). We conjecture

$$
\frac{(2 \pi)^{k-2} \Omega_{+}}{\Omega_{\infty}^{k-1}} \in O_{E_{\lambda}}^{\times} \text {and } \frac{(2 \pi)^{k-2} \Omega_{-}}{\Omega_{\infty}^{k-1}} \in O_{E_{\lambda}}^{\times}
$$

and hence $\mathcal{L}_{\Omega} \in \Lambda_{O_{E_{\lambda}}}(\mathcal{G})^{\times}$.

The difficulty in proving the above conjecture for $k \geq 3$ is due to the fact that it is not clear whether the motive $M(f)$ that it is attached to $f$ (a direct summand of the motive associated to a Kuga-Sato variety) coincides with the motive that we have associated to the Grössencharacter $\psi$ as $k-1$-fold tensor power of the elliptic curve associated to the character $\phi$ of type $(1,0)$. For a similar discussion see also [16, page 263].

We define

$$
\mathcal{L}_{f_{\psi}}^{\prime}:=\mathcal{L} \mathcal{L}_{\Omega}^{-1} \text { and } \mathcal{L}^{(t w)^{\prime}}{ }_{f_{\psi}}:=\mathcal{L}^{(t w)} \mathcal{L}_{\Omega}^{-1} .
$$

Assuming the above conjecture we have: 
Proposition 3.9. Let $M(f)^{\vee}$ be the dual motive to $M(f)$. Then $\mathcal{L}_{f_{\psi}}^{\prime}$ satisfies the following interpolation property for $0 \leq-j$ and $0<k-1+j$ :

$$
\begin{gathered}
\mathcal{L}_{f_{\psi}}^{\prime}\left(\rho \kappa^{j}\right)=\Gamma(k-1+j)^{d(\rho)} i^{d(\rho) j} \frac{L_{\{p\}}\left(M(f)^{\vee}, \rho, k-1+j\right)}{(2 \pi)^{2(k-2)+2 j} \Omega_{+}^{d^{+}(\rho)} \Omega_{-}^{d^{-}(\rho)}} e_{p}(\check{\rho}) \times \\
\frac{P_{p}\left(\stackrel{\vee}{\rho}, \frac{w}{p^{-j+1}}\right)}{P_{p}\left(\rho, w^{-1} p^{-j}\right)}\left(\frac{w}{p}\right)^{\mathfrak{f}_{p}(\check{\rho})} p^{j \mathfrak{f}_{p}(\check{\rho})}
\end{gathered}
$$

for all Artin representations $\rho$ of $\mathcal{G}$.

Proof. As in the proof in the elliptic curve case we consider two cases of artin representations, those that are one-dimensional and those that are induced from a character from $K$ to $\mathbb{Q}$. We explain here the case where $\rho=I n d \chi$ to indicate the similarities and differences with above. We compute

$$
\begin{aligned}
& \mathcal{L}\left(\rho \kappa^{j}\right)=\mathcal{L}_{\bar{\psi}}\left(\chi \kappa^{j}\right) \mathcal{L}_{\bar{\psi}}\left(\chi^{c} \kappa^{j}\right)= \\
& \Gamma(k-1+j)^{2} i^{2 j} \frac{L(\bar{\psi} \chi, k-1+j) L\left(\bar{\psi} \chi^{c}, k-1+j\right)}{(2 \pi)^{2 j} \Omega_{\infty}^{2(k-1)}} e_{\mathfrak{p}}(\bar{\chi}) e_{\overline{\mathfrak{p}}}(\bar{\chi}) \times \\
& \left(\frac{\psi(\mathfrak{p}) p^{j}}{p}\right)^{\mathfrak{f}_{\mathfrak{p}}(\bar{\chi})+\mathfrak{f}_{\bar{p}}(\bar{\chi})} P_{\mathfrak{p}}\left(\bar{\chi}, \frac{w}{p^{-j+1}}\right) P_{\overline{\mathfrak{p}}}\left(\chi, u^{-1} p^{-j}\right) P_{\bar{p}}\left(\bar{\chi}, \frac{w}{p^{-j+1}}\right) P_{\mathfrak{p}}\left(\chi, u^{-1} p^{-j}\right)= \\
& \Gamma(k-1+j)^{2} i^{2 j} \frac{L_{\{\mathfrak{p}, \bar{p}\}}(\bar{\psi} \chi, k-1+j) L_{\{\mathfrak{p}, \overline{\mathfrak{p}}\}}\left(\bar{\psi} \chi^{c}, k-1+j\right)}{(2 \pi)^{2 j} \Omega_{\infty}^{2(k-1)}} e_{\mathfrak{p}}(\bar{\chi}) e_{\overline{\mathfrak{p}}}(\bar{\chi}) \times \\
& \left(\frac{\psi(\mathfrak{p}) p^{j}}{p}\right)^{\mathfrak{f}_{\mathfrak{p}}(\bar{\chi})+\mathfrak{f}_{\overline{\mathfrak{p}}}(\bar{\chi})} \frac{P_{\mathfrak{p}}\left(\bar{\chi}, \frac{w}{p^{-j+1}}\right) P_{\overline{\mathfrak{p}}}\left(\chi, u^{-1} p^{-j}\right) P_{\overline{\mathfrak{p}}}\left(\bar{\chi}, \frac{w}{p^{-j+1}}\right) P_{\mathfrak{p}}\left(\chi, u^{-1} p^{-j}\right)}{P_{\mathfrak{p}}\left(\chi, w^{-1} p^{-j}\right) P_{\overline{\mathfrak{p}}}\left(\chi, u^{-1} p^{-j}\right) P_{\overline{\mathfrak{p}}}\left(\chi, w^{-1} p^{-j}\right) P_{\mathfrak{p}}\left(\chi, u^{-1} p^{-j}\right)}= \\
& \Gamma(k-1+j)^{2} i^{2 j} \frac{L_{\{\mathfrak{p}, \overline{\mathfrak{p}}\}}(\bar{\psi} \chi, k-1+j) L_{\{\mathfrak{p}, \overline{,}\}}\left(\bar{\psi} \chi^{c}, k-1+j\right)}{(2 \pi)^{2 j} \Omega_{\infty}^{2(k-1)}} e_{\mathfrak{p}}(\bar{\chi}) e_{\overline{\mathfrak{p}}}(\bar{\chi}) \times \\
& \left(\frac{\psi(\mathfrak{p}) p^{j}}{p}\right)^{\mathfrak{f}_{\mathfrak{p}}(\bar{\chi})+\mathfrak{f}_{\bar{p}}(\bar{\chi})} \frac{P_{\mathfrak{p}}\left(\bar{\chi}, \frac{w}{p^{-j+1}}\right) P_{\overline{\mathfrak{p}}}\left(\bar{\chi}, \frac{w}{p^{-j+1}}\right)}{P_{\mathfrak{p}}\left(\chi, w^{-1} p^{-j}\right) P_{\overline{\mathfrak{p}}}\left(\chi, w^{-1} p^{-j}\right)}= \\
& e_{p}(\check{\rho})\left(\frac{\psi(\mathfrak{p}) p^{j}}{p}\right)^{\mathfrak{f}_{p}(\rho)} \Gamma(k-1+j)^{2} i^{2 j} \frac{L_{\{p\}}\left(M(f)^{\vee}, \rho, k-1+j\right)}{(2 \pi)^{2 j} \Omega_{\infty}^{2(k-1)}} \frac{P_{p}\left(\check{\rho}, \frac{w}{p^{-j+1}}\right)}{P_{p}\left(\rho, w^{-1} p^{-j}\right)} .
\end{aligned}
$$

The last equation follows from the fact that since $\psi$ induces the cusp form $f_{\psi}=f=$ $\sum_{n \geq 1} a_{n} q^{n}$, the character $\bar{\psi}$ induces a cuspidal newform $f_{\bar{\psi}}=\sum_{n \geq 1} \overline{a_{n}} q^{n}$ where the complex conjugation on the coefficients $a_{n}$ is with respect to our fixed embedding $F \hookrightarrow \overline{\mathbb{Q}}$. Actually one has that $\overline{a_{n}}=\epsilon_{f}^{-1}(n) a_{n}$. But then $f_{\bar{\psi}}$ corresponds to the 
dual motive $M(f)^{\vee}$. Then as above we conclude that $\mathcal{L}_{f_{\psi}}^{\prime}=\mathcal{L} \mathcal{L}_{\Omega}^{-1}$ has the claimed interpolation property. $\square$

Similarly we have

Proposition 3.10. The measure $\mathcal{L}^{(t w)^{\prime}}{ }_{f_{\psi}}$ satisfies the following interpolation property for $0 \leq-j$ and $0<k-1+j$,

$$
\mathcal{L}^{(t w)^{\prime}}{ }_{f_{\psi}}\left(\rho \kappa^{j}\right)=\Gamma(1-j)^{d(\rho)} i^{d(\rho) j} \frac{L_{\{p\}}(M(f), \check{\rho}, 1-j)}{(2 \pi)^{-2 j} \Omega_{+}^{d^{+}(\rho)} \Omega_{-}^{d^{-}(\rho)}} e_{p}(\rho) \frac{P_{p}\left(\rho, u^{-1} p^{-j}\right)}{P_{p}\left(\check{\rho}, \frac{u}{p^{-j+1}}\right)}\left(u p^{j}\right)^{-f_{p}(\check{\rho})}
$$

for all Artin representations $\rho$ of $\mathcal{G}$.

Proof. As above we have

$$
\begin{aligned}
& \mathcal{L}^{(t w)}\left(\rho \kappa^{j}\right)=\mathcal{L}_{\bar{\psi}}^{(t w)}\left(\chi \kappa^{j}\right) \mathcal{L}_{\bar{\psi}}^{(t w)}\left(\chi^{c} \kappa^{j}\right)= \\
& \Gamma(k-1+j)^{2} i^{2 j} \frac{L(\psi \bar{\chi}, 1-j) L\left(\psi \bar{\chi}^{c}, 1-j\right)}{(2 \pi)^{2 j} \Omega_{\infty}^{2(k-1)}} e_{\mathfrak{p}}(\chi) e_{\overline{\mathfrak{p}}}(\chi)\left(u p^{j}\right)^{-\mathfrak{f}_{\mathfrak{p}}(\bar{\chi})-\mathfrak{f}_{\overline{\mathfrak{p}}}(\bar{\chi})} \\
& \times P_{\mathfrak{p}}\left(\bar{\chi}, \frac{w}{p^{-j+1}}\right) P_{\overline{\mathfrak{p}}}\left(\chi, u^{-1} p^{-j}\right) P_{\overline{\mathfrak{p}}}\left(\bar{\chi}, \frac{w}{p^{-j+1}}\right) P_{\mathfrak{p}}\left(\chi, u^{-1} p^{-j}\right)\left(\frac{\Gamma_{\psi}(1-j)}{\Gamma_{\psi}(k-1+j)}\right)^{2}= \\
& \Gamma(k-1+j)^{2} i^{2 j} \frac{L_{\{\mathfrak{p}, \overline{\mathfrak{p}}\}}(\psi \bar{\chi}, 1-j) L_{\{\mathfrak{p}, \overline{\mathfrak{p}}\}}\left(\psi \bar{\chi}^{c}, 1-j\right)}{(2 \pi)^{2 j} \Omega_{\infty}^{2(k-1)}} e_{\mathfrak{p}}(\chi) e_{\overline{\mathfrak{p}}}(\chi)\left(u p^{j}\right)^{-\mathfrak{f}_{\mathfrak{p}}(\bar{\chi})-\mathfrak{f}_{\bar{p}}(\bar{\chi})} \times \\
& \frac{P_{\mathfrak{p}}\left(\bar{\chi}, \frac{w}{p^{-j+1}}\right) P_{\overline{\mathfrak{p}}}\left(\chi, u^{-1} p^{-j}\right) P_{\overline{\mathfrak{p}}}\left(\bar{\chi}, \frac{w}{p^{-j+1}}\right) P_{\mathfrak{p}}\left(\chi, u^{-1} p^{-j}\right)}{P_{\mathfrak{p}}\left(\bar{\chi}, \frac{w}{p^{-j+1}}\right) P_{\overline{\mathfrak{p}}}\left(\bar{\chi}, \frac{u}{p^{-j+1}}\right) P_{\overline{\mathfrak{p}}}\left(\bar{\chi}, \frac{w}{p^{-j+1}}\right) P_{\mathfrak{p}}\left(\bar{\chi}, \frac{u}{p^{-j+1}}\right)}\left(\frac{\Gamma_{\psi}(1-j)}{\Gamma_{\bar{\psi}}(k-1+j)}\right)^{2}= \\
& \Gamma(k-1+j)^{2} i^{2 j} \frac{L_{\{\mathfrak{p}, \overline{\mathfrak{p}}\}}(\psi \bar{\chi}, 1-j) L_{\{\mathfrak{p}, \overline{\mathfrak{p}}\}}\left(\psi \bar{\chi}^{c}, 1-j\right)}{(2 \pi)^{2 j} \Omega_{\infty}^{2(k-1)}} e_{\mathfrak{p}}(\chi) e_{\overline{\mathfrak{p}}}(\chi)\left(u p^{j}\right)^{-\mathfrak{f}_{\mathfrak{p}}(\bar{\chi})-\mathfrak{f}_{\overline{\mathfrak{p}}}(\bar{\chi})} \\
& \times \frac{P_{\mathfrak{p}}\left(\chi, u^{-1} p^{-j}\right) P_{\overline{\mathfrak{p}}}\left(\chi, u^{-1} p^{-j}\right)}{P_{\mathfrak{p}}\left(\bar{\chi}, \frac{u}{p^{-j+1}}\right) P_{\overline{\mathfrak{p}}}\left(\bar{\chi}, \frac{u}{p^{-j+1}}\right)}\left(\frac{\Gamma_{\psi}(1-j)}{\Gamma_{\bar{\psi}}(k-1+j)}\right)^{2}= \\
& e_{p}(\rho)\left(u p^{j}\right)^{-f_{p}(\breve{\rho})} \Gamma(k-1+j)^{2} i^{2 j} \frac{L_{\{p\}}(M(f), \check{\rho}, 1-j)}{(2 \pi)^{2 j} \Omega_{\infty}^{2(k-1)}} \frac{P_{p}\left(\rho, u^{-1} p^{-j}\right)}{P_{p}\left(\check{\rho}, \frac{u}{p^{-j+1}}\right)}\left(\frac{\Gamma_{\psi}(1-j)}{\Gamma_{\bar{\psi}}(k-1+j)}\right)^{2} .
\end{aligned}
$$

We recall that $\Gamma_{\phi}(s)=\frac{\Gamma(s-\min (k, j))}{(2 \pi)^{s-m i n(k, j)}}$ for $\phi$ a Grössencharacter of type $(k, j)$. In particular for the character $\psi$ of type $(k-1,0)$ (hence $\bar{\psi}$ of type $(0, k-1))$ we have that

$$
\frac{\Gamma_{\psi}(1-j)}{\Gamma_{\bar{\psi}}(k-1+j)}=\frac{\Gamma(1-j)}{\Gamma(k-1+j)}(2 \pi)^{k-2+2 j} .
$$

This allows us to conclude the proof of the proposition. $\mathbf{C}$

Altogether we obtain the following (under our assumption over the torsion part of $G$ ),

TheOrem 3.11. Assuming Conjectures 3.2 and 3.8 there exists $\mathcal{L}_{M(f)}^{\prime} \in$ $K_{1}\left(\Lambda_{D}(\mathcal{G})_{S}\right)$ satisfying Conjecture 3.3 and 3.4. 
4. Appendix: Galois descent for Iwasawa algebras. The aim of this appendix is to provide some results for Galois descent of (non-commutative) $p$-adic $L$-functions, by which we mean the following general question:

Assume that $G$ is a compact $p$-adic Lie group which possesses an closed normal subgroup $H$ such that $G / H=: \Gamma \cong \mathbb{Z}_{p}$ and let $\mathcal{O}=\mathcal{O}_{L}$ denote the ring of integers of a finite extension $L$ over $\mathbb{Q}_{p}$. Then we denote by $D$ the discrete valuation ring of the completion $\tilde{L}$ of the maximal unramified extension $L^{n r}$ of $L$. If $\mathcal{L} \in K_{1}\left(\Lambda_{D}(G)_{S^{*}}\right)$ has the property

$$
\mathcal{L}(\rho) \in \mathcal{O}(\rho) \text { for all } \rho \in \operatorname{Irr} G,
$$

does it hold that there exists an element $\mathcal{L}^{\prime} \in K_{1}\left(\Lambda_{\mathcal{O}}(G)_{S^{*}}\right)$ with

$$
\mathcal{L}^{\prime}(\rho)=\mathcal{L}(\rho) \text { for all } \rho \in \operatorname{IrrG} ?
$$

Here $\operatorname{Irr} G$ denotes the set of isomorphism classes of irreducible Artin representations of $G$ with values in $\overline{\mathbb{Q}_{p}}$ while for every $\rho \in \operatorname{Irr} G$ we write $\mathcal{O}(\rho)$ for the discrete valuation ring of $L(\rho)=\overline{\mathbb{Q}}_{p}{ }^{G_{L, \rho}}$, where $G_{L, \rho}$ is the stabilizer subgroup of $\rho$ in $G_{L}=G\left(\overline{\mathbb{Q}_{p}} / L\right)$ with respect to the natural action on the coefficients of $\rho$.

We start with a simple observation in the abelian case:

LEMma 4.1. Let $G$ be a topological finitely generated virtual pro-p, abelian group, such that the exponent of its torsion part divides $p-1$. Assume that for a given $f \in \Lambda_{D}(G)$ the values $f(\rho)$ belongs to $L(\rho)$ (and hence to $\mathcal{O}(\rho)$ ), for all (irreducible) Artin characters $\rho$ of $G$. Then $f$ is already contained in $\Lambda_{\mathcal{O}}(G)$.

Proof. With out loss of generality me may assume that $G \cong \mathbb{Z}_{p}^{d}$ for some natural number $d$, because otherwise me can decompose $\Lambda_{D}(G)$ into a product of such rings by our assumption on the torsion part of $G$. Also by taking inverse limits afterwards we may and do assume that $G$ is a finite $p$-group. Then it is well-known (e.g. a variant of prop. 4.5.14 in [28]) that we have a $G_{L}$-invariant isomorphism

$$
\phi: \mathbb{C}_{p}[G] \cong \operatorname{Hom}\left(R(G), \mathbb{C}_{p}\right), \quad f=\sum m_{g} g \mapsto\left(\rho \mapsto f(\rho)=\sum m_{g} \rho(g)\right),
$$

where on the left hand side the Galois action is just on the coefficients while on the right hand side $h^{g}(\rho)=g h\left(g^{-1} \rho\right)$ for all $g \in G_{\mathbb{Q}_{p}}$, and $h$ any homomorphism from the group of virtual representations $R(G)$ of $G$ defined over $\overline{\mathbb{Q}_{p}}$. Recall that by the Ax-Sen-Tate theorem

$$
\mathbb{C}_{p}^{H}=\tilde{L}
$$

for $\left.H:=G\left(\overline{\mathbb{Q}_{p}}\right) / L_{p}^{n r}\right)$. Similarly, if $H_{\rho}$ denotes the stabiliser of $\rho$ we get

$$
\tilde{L}(\rho):=\mathbb{C}_{p}^{H_{\rho}}=\widehat{{\overline{\mathbb{Q}_{p}}}^{H_{\rho}}}=\widehat{L^{n r}(\rho)} .
$$

Taking $H$ - and $G_{L}$-invariants of $\phi$ thus induces the following commutative diagram

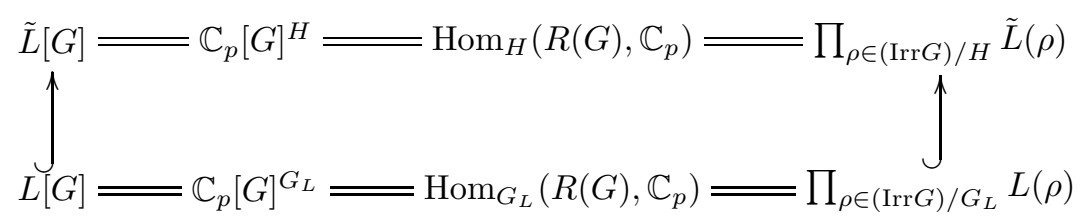


where $(\operatorname{Irr} G) / U$ denotes the Galois-orbits with respect to some closed subgroup $U \subseteq$ $G_{L}$. Since $L(\rho) / L$ is totally ramified, it is easy to see that

$$
(\operatorname{Irr} G) / H=(\operatorname{Irr} G) / G_{L} .
$$

Now, by assumption $f \in D[G] \subseteq \tilde{L}[G]$ satisfies $f(\rho) \in \mathcal{L}(\rho)$ for all $\rho \in \operatorname{Irr} G$, i.e. $\phi(f) \in \prod_{\rho \in(\operatorname{Irr} G) / G_{L}} L(\rho)$. Hence $f \in L[G] \cap D[G]=\mathcal{O}[G]$, because $L \cap D=\{x \in$ $\left.\left.L|| x\right|_{p} \leq 1\right\}=\mathcal{O}$ coefficientwise.

In the following we will use Fröhlich's Hom-description as it has been adapted to Iwasawa theory by Ritter and Weiss $[20, \S 3]$. We have the following commutative diagram

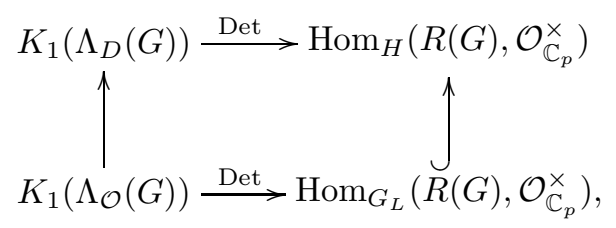

where the homomorphism Det is defined as follows: for $\rho \in \operatorname{Irr} G$ we obtain a homomorphism of rings

$$
\rho: \Lambda_{D}(G) \rightarrow M_{n_{\rho}}\left(\overline{\mathbb{Z}_{p}}\right),
$$

taking $K_{1}(-)$ of which gives a group homomorphism

$$
\psi_{\rho}: K_{1}\left(\Lambda_{D}(G)\right) \rightarrow K_{1}\left(M_{n_{\rho}}\left(\overline{\mathbb{Z}_{p}}\right)\right) \cong K_{1}\left(\overline{\mathbb{Z}_{p}}\right) \subseteq \mathcal{O}_{\mathbb{C}_{p}}^{\times} .
$$

Now we set $\operatorname{Det}(\mathcal{L})(\rho):=\psi_{\rho}(\mathcal{L})$.

Setting $\Delta:=G\left(L^{n r} / L\right)$ we obtain the following generalisation of a theorem of M. Taylor [30, §8, thm. 1.4]:

TheOREM 4.2. For $\mathcal{O}$ unramified over $\mathbb{Z}_{p}$ we have

$$
\operatorname{Det}\left(K_{1}\left(\Lambda_{D}(G)\right)\right)^{\Delta}=\operatorname{Det}\left(K_{1}\left(\Lambda_{\mathcal{O}}(G)\right)\right) .
$$

Proof. In Taylor's theorem (loc. cit.) $G$ is finite and $D$ is the valuation ring of a finite unramified extension of $L$. His proof generalizes immediately to the case of our more general $D$, see [15] for details, thus we only have to show how the general case can be reduced to the case of finite groups. To this end write $G=\underset{n}{\lim } G_{n}$ as inverse limit of finite groups. By Taylor's result we have compatible continuous maps

$$
\mathcal{O}\left[G_{n}\right]^{\times} \stackrel{\operatorname{Det}}{\longrightarrow} \operatorname{Det}\left(K_{1}\left(\Lambda_{D}\left(G_{n}\right)\right)\right)^{\Delta} \longleftrightarrow \operatorname{Hom}_{H}\left(R\left(G_{n}\right), \mathcal{O}_{\mathbb{C}_{p}}^{\times}\right)^{\Delta}
$$

where the topology on $\operatorname{Hom}_{H}\left(R\left(G_{n}\right), \mathcal{O}_{\mathbb{C}_{p}}^{\times}\right) \cong \prod_{\rho \in \operatorname{Irr} G_{n} / H}\left(\mathcal{O}_{\mathbb{C}_{p}}^{\times}\right)^{H_{\rho}}$ is induced from the valuation topology on $\mathbb{C}_{p}$. Taking the inverse limit yields, by the compactness of $\Lambda_{\mathcal{O}}(G)^{\times}=\underset{n}{\lim }\left(\mathcal{O} / \pi^{n}\left[G_{n}\right]\right)^{\times}$and by letting $R(G):=\underset{n}{\lim } R\left(G_{n}\right)$ denote the free abelian group on the isomorphism classes of irreducible Artin representations of $G$, a factorization of the homomorphism Det into

$$
\Lambda_{\mathcal{O}}(G)^{\times} \underset{\operatorname{Det}}{\longrightarrow}\left(\lim _{n} \operatorname{Det}\left(K_{1}\left(\Lambda_{D}\left(G_{n}\right)\right)\right)\right)^{\Delta} \longleftrightarrow \operatorname{Hom}_{G_{L}}\left(R(G), \mathcal{O}_{\mathbb{C}_{p}}^{\times}\right) .
$$


The claim follows because denoting by res $_{n}: \operatorname{Hom}_{H}\left(R(G), \mathcal{O}_{\mathbb{C}_{p}}^{\times}\right) \quad \rightarrow$ $\operatorname{Hom}_{H}\left(R\left(G_{n}\right), \mathcal{O}_{\mathbb{C}_{p}}^{\times}\right)$the restriction we obtain from the universal mapping property for

$$
\overleftarrow{\lim }_{n} \operatorname{Hom}_{H}\left(R\left(G_{n}\right), \mathcal{O}_{\mathbb{C}_{p}}^{\times}\right) \cong \operatorname{Hom}_{H}\left(R(G), \mathcal{O}_{\mathbb{C}_{p}}^{\times}\right)
$$

the inclusions

$$
\begin{aligned}
\operatorname{Det}\left(K_{1}\left(\Lambda_{D}(G)\right)\right) & \subseteq \underset{n}{\operatorname{limim}_{n}}\left(\text { res }_{n} \circ \operatorname{Det}\right) \\
& \subseteq \underset{\lim _{n}}{\lim } \operatorname{Det}\left(K_{1}\left(\Lambda_{D}\left(G_{n}\right)\right)\right),
\end{aligned}
$$

whence the obvious inclusion

$$
\operatorname{Det}\left(K_{1}\left(\Lambda_{\mathcal{O}}(G)\right)\right) \subseteq \operatorname{Det}\left(K_{1}\left(\Lambda_{D}(G)\right)\right)^{\Delta} \subseteq\left(\overleftarrow{n}_{n}^{\lim } \operatorname{Det}\left(K_{1}\left(\Lambda_{D}\left(G_{n}\right)\right)\right)\right)^{\Delta}
$$

is surjective.

There are (at least) two obvious questions: Firstly whether this descent result does also hold for the full $K_{1}$-groups ( this amounts to an analogous statement for the $S K_{1}$-terms) and secondly whether the analogue for the localisations holds, too. We shall at least show a weak version towards the second issue. To this end we give a variant of Ritter and Weiss' Hom-description: Consider the following commutative diagram

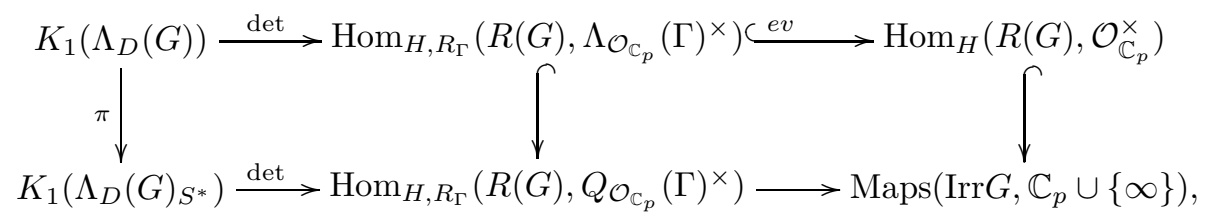

where

- $Q_{\mathcal{O}_{\mathbb{C}_{p}}}(\Gamma)$ denotes the quotient field of $\Lambda_{\mathcal{O}_{\mathbb{C}_{p}}}(\Gamma)$,

- for $A$ either $\Lambda_{\mathcal{O}_{\mathbb{C}_{p}}}(\Gamma)^{\times}$or $Q_{\mathcal{O}_{\mathbb{C}_{p}}}(\Gamma)^{\times}$we denote by $\operatorname{Hom}_{H, R_{\Gamma}}(R(G), A)$ the both $H$-invariant and $R(\Gamma)$-twist-invariant homomorphisms. The latter means that $f(\rho \otimes \chi)=\operatorname{tw}_{\chi^{-1}}(f(\rho))$ for all $\chi$ in $R(\Gamma)$; here $\chi$ is considered as element in $R(G)$ via the fixed surjection $G \rightarrow \Gamma$ and $\operatorname{tw}_{\chi}: A \rightarrow A$ is induced by the action $\gamma \mapsto \chi\left(\gamma^{-1}\right) \gamma$ on $\Lambda_{\mathcal{O}_{\mathbb{C}_{p}}}(\Gamma)$, compare with [20, thm. 8],

- - analogously as for Det - the homomorphisms det are induced from the ring homomorphisms

$$
\Lambda_{D}(G) \rightarrow M_{n_{\rho}}\left(\mathcal{O}_{\mathbb{C}_{p}}\right) \widehat{\otimes}_{D} \Lambda_{D}(\Gamma) \cong M_{n_{\rho}}\left(\Lambda_{\mathcal{O}_{\mathbb{C}_{p}}}(\Gamma)\right), g \mapsto \rho(g) \otimes \bar{g},
$$

and its localisation at $S^{*}$

$$
\Lambda_{D}(G)_{S^{*}} \rightarrow M_{n_{\rho}}\left(\Lambda_{\mathcal{O}_{\mathbb{C}_{p}}}(\Gamma)\right)_{S^{*}} \cong M_{n_{\rho}}\left(Q_{\mathcal{O}_{\mathbb{C}_{p}}}(\Gamma)\right),
$$

respectively. 
- the homomorphism ev $: \operatorname{Hom}_{H, R_{\Gamma}}\left(R(G), \Lambda_{\mathcal{O}_{\mathbb{C}_{p}}}(\Gamma)^{\times}\right) \rightarrow \operatorname{Hom}_{H}\left(R(G), \mathcal{O}_{\mathbb{C}_{p}}^{\times}\right)$, which is induced by the augmentation map $\Lambda_{\mathcal{O}_{\mathbb{C}_{p}}}(\Gamma)^{\times} \rightarrow \mathcal{O}_{\mathbb{C}_{p}}^{\times}$, i.e. $\operatorname{ev}(f)(\rho)=$ $f(\rho)\left(\chi_{\text {triv }}\right)$, where the latter means evaluation at the trivial character $\chi_{\text {triv }}$ of $\Gamma$, is injective by the Weierstrass preparation theorem and the twist invariance of $f$ (in the kernel of $e v$ ):

$$
f(\rho)(\chi)=\operatorname{tw}_{\chi^{-1}}(f(\rho))\left(\chi_{\text {triv }}\right)=f(\rho \otimes \chi)\left(\chi_{\text {triv }}\right)=1
$$

for all $\chi$, whence $f(\rho)=1$ for all $\rho$,

- we do not know whether the map $\operatorname{Hom}_{H, R_{\Gamma}}\left(R(G), Q_{\mathcal{O}_{\mathbb{C}_{p}}}(\Gamma)^{\times}\right) \rightarrow$ $\operatorname{Maps}\left(\operatorname{Irr} G, \mathbb{C}_{p} \cup\{\infty\}\right)$ is injective.

Note that

- a similar diagram exists with $\mathcal{O}$-coefficients (instead of $D$ ) and $H$ replaced by $G_{L}$ and that by construction the morphisms det commute with the canonical change of coefficients maps.

- the composition of the top-line of the above diagram equals Det.

- the image of $\mathcal{L}$ in $\operatorname{Maps}\left(\operatorname{Irr} G, \mathbb{C}_{p} \cup\{\infty\}\right)$ is the map which attaches to $\rho$ the value $\mathcal{L}(\rho)$ of $\mathcal{L}$ at $\rho$ and the map into this target is multiplicative at least in the following sense: if $f(\rho) \neq 0, \infty$ for all $\rho$, then $(g f)(\rho)=g(\rho) f(\rho)$ for all $\rho$ (with $\infty \cdot a=a \cdot \infty=\infty$ and $0 \cdot a=a \cdot 0=0$ for $a \neq 0, \infty$ ).

By $\iota$ we denote the canonical map $K_{1}\left(\Lambda_{\mathcal{O}}(G)_{S^{*}}\right) \rightarrow K_{1}\left(\Lambda_{D}(G)_{S^{*}}\right)$.

TheOREM 4.3. Assume that

(i) $\mathcal{O}$ is absolutely unramified,

(ii) $L(\rho) / L$ is totally ramified (or trivial) for all $\rho \in \operatorname{Irr} G$,

(iii) $\mathcal{L} \in K_{1}\left(\Lambda_{D}(G)_{S^{*}}\right)$ is induced from an element in $\Lambda_{D}(G) \cap\left(\Lambda_{D}(G)_{S^{*}}\right)^{\times}$

(iv) $\mathcal{L}$ satisfies

$$
\mathcal{L}(\rho) \in L(\rho) \text { for all } \rho \in \operatorname{Irr} G,
$$

(v) there is an $F \in K_{1}\left(\Lambda_{\mathcal{O}}(G)_{S^{*}}\right)$ such that $\partial\left(\mathcal{L} \cdot \iota(F)^{-1}\right)=0$ (e.g. if $\mathcal{L}$ is the characteristic element of the base change from a module in $\mathfrak{M}_{\mathcal{O}, H}(G)$ ).

Then there exists $\mathcal{L}^{\prime} \in K_{1}\left(\Lambda_{\mathcal{O}}(G)_{S^{*}}\right)$ with

$$
\mathcal{L}^{\prime}(\rho)=\mathcal{L}(\rho) \text { for all } \rho \in \operatorname{IrrG}
$$

and

$$
\partial\left(\mathcal{L}^{\prime}\right)=\partial(F),
$$

in particular

$$
\iota \partial\left(\mathcal{L}^{\prime}\right)=\partial(\mathcal{L}) .
$$

Proof. Consider the following commutative diagram with exact rows

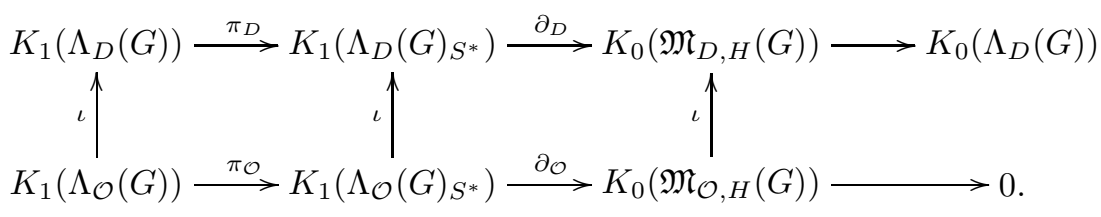


By assumption there exists $\mathcal{D} \in K_{1}\left(\Lambda_{D}(G)\right)$ such that $\pi_{D}(\mathcal{D})=\mathcal{L} \cdot \iota(F)^{-1}$. By Lemma 4.4 below $\operatorname{Det}(\mathcal{D})$ belongs to $\operatorname{Det}\left(K_{1}\left(\Lambda_{D}(G)\right)\right)^{\Delta}=\operatorname{Det}\left(K_{1}\left(\Lambda_{\mathcal{O}}(G)\right)\right)$, i.e. there is a $\mathcal{D}^{\prime} \in K_{1}\left(\Lambda_{\mathcal{O}}(G)\right)$ such that $\mathcal{D}(\rho)=\mathcal{D}^{\prime}(\rho)$ for all $\rho$ in $R(G)$. Setting $\mathcal{L}^{\prime}:=\pi_{\mathcal{O}}(F) \mathcal{D}^{\prime}$ and recalling that $\mathcal{D}^{\prime}(\rho)=\mathcal{D}(\rho) \neq 0, \infty$ we calculate

$$
\begin{aligned}
\mathcal{L}^{\prime}(\rho) & =F(\rho) \mathcal{D}^{\prime}(\rho) \\
& =F(\rho) \mathcal{D}(\rho) \\
& =\operatorname{det}(F)(\rho)\left(\chi_{\text {triv }}\right) \cdot \operatorname{det}(\mathcal{D})(\rho)\left(\chi_{\text {triv }}\right) \\
& =(\operatorname{det}(F)(\rho) \operatorname{det}(\mathcal{D})(\rho))\left(\chi_{\text {triv }}\right) \\
& =\operatorname{det}(\iota(F) \pi(\mathcal{D}))(\rho)\left(\chi_{\text {triv }}\right) \\
& =\operatorname{det}(\mathcal{L})(\rho)\left(\chi_{\text {triv }}\right) \\
& =\mathcal{L}(\rho),
\end{aligned}
$$

whence the theorem is proven.

LEMma 4.4. With notation as in the previous proof we have

(i) $\operatorname{det}(\mathcal{D})(\rho) \in \mathcal{O}(\rho)[[\Gamma]]$ for all $\rho \in R(G)$,

(ii) $\mathcal{D}(\rho)=\operatorname{Det}(\mathcal{D})(\rho) \in \mathcal{O}(\rho)$ for all $\rho \in R(G)$,

(iii) $\operatorname{Det}(\mathcal{D}) \in \operatorname{Det}\left(K_{1}\left(\Lambda_{D}(G)\right)\right)^{\Delta}$.

Proof. By construction and assumption ( $\mathcal{L}$ being induced from ....) we have $\operatorname{det}(\mathcal{L})(\rho) \in D(\rho)[[\Gamma]]$ with $D(\rho):=\left(\mathcal{O}_{\mathbb{C}_{p}}\right)^{H_{\rho}}$ and thus by Lemma $4.1 \operatorname{det}(\mathcal{L})(\rho)$ belongs to $\mathcal{O}(\rho)[[\Gamma]]$. On the other hand $\operatorname{det}(F)(\rho)$ belongs to $Q_{\mathcal{O}(\rho)}(\Gamma)^{\times}$, whence

$$
\operatorname{det}(\mathcal{D})(\rho) \in Q_{\mathcal{O}(\rho)}(\Gamma)^{\times} \cap \Lambda_{D(\rho)}(\Gamma)^{\times} .
$$

We claim that this intersection equals $\Lambda_{\mathcal{O}(\rho)}(\Gamma)^{\times}$. One inclusion being obvious we assume that $q$ belongs to the intersection. It follows immediately that $q(\chi)$ belongs to $\mathcal{O}(\rho, \chi):=\left(\mathcal{O}_{\mathbb{C}_{p}}\right)^{G_{L, \rho} \cap G_{L, \chi}}$ for all $\chi \in R(\Gamma)$. Thus the claim follows Lemma 4.1 (with $\mathcal{O}(\rho)$ for the base ring $\mathcal{O}$ ). This proves (i) and as $\mathcal{D}(\rho)=\operatorname{det}(\mathcal{D})(\rho)\left(\chi_{\text {triv }}\right)$ also (ii) follows. In order to show (iii) observe first that $\Delta$ acts trivially on $\operatorname{Irr} G / G_{\tilde{L}}=$ $\operatorname{Irr} G / G_{L}$. Hence from the following commutative diagram the statement is clear:

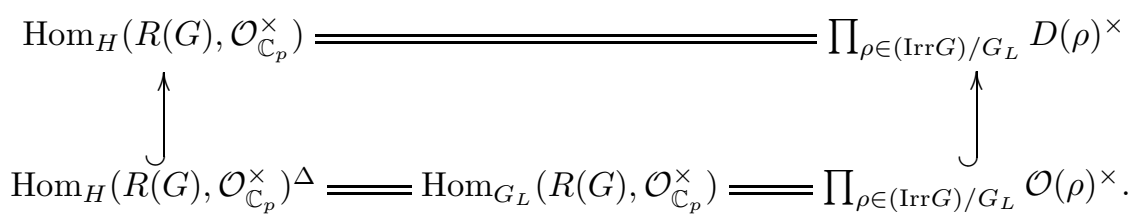

REFERENCES

[1] K. Ardakov and K. Brown, Primeness, Semiprimeness and localization in Iwasawa algebras, Transactions of the American Mathematical Society, 359:4 (2007), pp. 1499-1516.

[2] D. Bernardi, C. Goldstein and N. Stephens, Notes p-adiques sur les courbes elliptique, Journal für die reine und angewandte Mathematik, 351 (1984).

[3] D. Blasius, On the critical values of Hecke L-series, Annals of Mathematics, 124 (1986).

[4] D. Blasius, Period relations and critical values of L-functions, Pacific Journal of Mathematics, 181:3 (1997). 
[5] D. Burns and O. Venjakob, Leading terms on p-adic L-functions in non-commutative Iwasawa theory, Doc. Math. Extra Volume: John. H. Coates 60th Birthday (2006), pp. 165209.

[6] D. Burns and O. Venjakob, On descent theory and main conjectures in non-commutative Iwasawa theory, J. Inst. Math. Jussieu, 10:1 (2010), pp. 59-118.

[7] J. Coates, T. Fukaya, K. Kato, R. Sujatha, and O. Venjakob, The $G L_{2}$ main conjecture for elliptic curves without complex multiplication, Publ. Math. IHES., 101:1 (2005), pp. 163-208.

[8] P. Deligne, Valeurs de fonctions L et périoded d'intégrales, In Proceedings of Symposia in Pure Mathematics, 33 (1979).

[9] E. DE Shalit, Iwasawa theory of elliptic curves with complex multiplication, Perspectives in mathematics, vol. 3, Academic Press, 1987.

[10] J.-M. Fontaine, Représentations p-adiques semi-stables, Astérisque, 233 (1994), pp. 113-184.

[11] T. Fukaya AND K. Kato, A formulation of conjectures on p-adic zeta functions in noncommutative Iwasawa theory, Proceedings of the St. Petersburg Mathematical Society, Vol. XII (Providence, RI), Amer. Math. Soc. Transl. Ser. 2, vol. 219, Amer. Math. Soc., 2006, pp. $1-86$.

[12] R. Gillard, Remarques sur les Unités Cyclotomiques et sur les Unités Elliptiques, Journal of Number Theory, 11 (1979), pp. 21-48.

[13] R. Gillard and G. Robert, Groupe des unités elliptiques, Bulletin de la S.M.F., 107 (1979), pp. 305-317.

[14] Y. Hatchimori and O. Venjakob, Completely faithful Selmer groups over Kummer extensions, Doc. Math. Extra Volume: K. Kato 50th Birthday (2003), pp. 443-478.

[15] D. IZyChev and O. Venjakob, Galois invariants of $K_{1}$-groups of Iwasawa algebras, preprint (2010), http://arxiv.org/abs/1006.5357.

[16] K. KATO, p-adic Hodge theory and values of zeta functions of modular forms, Astérique, 295 (2004), pp. 117-290.

[17] N. KATZ, p-adic interpolation properties of real analytic Eisenstein series, Annals of Mathematics, 104 (1976), pp. 459-571.

[18] B. PERrin-Riou, Groupe de Selmer d'une courbe elliptique à multiplication complexe, Compositio Mathematica, 43:3 (1981), pp. 387-417.

[19] K. RiBET, Galois representations attached to eigenforms of nebentypus, In Modular Functions of one Variable V, Lecture Notes in Mathematics, Vol. 601, Springer 1977.

[20] J. Ritter And A. Weiss, Toward equivariant Iwasawa theory, II, Indag. Mathem. N.S., 15:4 (2004), pp. 549-572.

[21] K. RuBin, The "main conjectures" of Iwasawa theory for imaginary quadratic fields., Invent. Math., 103 (1991), pp. 25-68.

[22] N. Schappacher, Periods of Hecke Characters, Lecture Notes in Mathematics 1301, SpringerVerlag 1988.

[23] P. Schneider and O. Venjakob, Localizations and completions of skew power series rings, Am. J. Math. 132:1 (2010), pp. 1-36.

[24] L. Schneps, On the $\mu$-invariant of $p$-adic L-functions attached to elliptic curves with complex multiplication, Journal of Number Theory, 25 (1987), pp. 20-33.

[25] J.-P. SerRe, Linear Representation of Finite Groups, Graduate Texts in Mathematics 42, Springer (1977).

[26] J. H. Silverman, The arithmetic of elliptic curves, Graduate Texts in Mathematics, vol. 106, Springer-Verlag, New York, 1986.

[27] J. H. Silverman, Advanced topics in the arithmetic of elliptic curves, Graduate Texts in Mathematics, vol. 151, Springer-Verlag, New York, 1994.

[28] V. P. Snaith, Explicit Brauer induction, Cambridge Studies in Advanced Mathematics, vol. 40, Cambridge University Press, Cambridge, 1994, with applications to algebra and number theory.

[29] J. Tate, Number Theoretic Background, Proceedings of Symposia in Pure Mathematics, 33 (1979).

[30] M. TAYLOR, Classgroups of group rings, London Mathematical Society Lecture Note Series, vol. 91, Cambridge University Press, Cambridge, 1984.

[31] O. Venjakob, Characteristic Elements in Noncommutative Iwasawa Theory, Habilitationsschrift, Ruprecht-Karls-Universität Heidelberg (2003).

[32] R. YAGER, On the two variable p-adic L-functions, Annals of Mathematics, 115 (1982), pp. 411449. 